\title{
The QCD spectrum with three quark flavors
}

\author{
Claude Bernard \\ Department of Physics, Washington University, St. Louis, MO 63130, USA \\ Tom Burch, Kostas Orginos* and Doug Toussaint \\ Department of Physics, University of Arizona, Tucson, AZ 85721, USA \\ Thomas A. DeGrand \\ Physics Department, University of Colorado, Boulder, CO 80309, USA \\ Carleton DeTar \\ Physics Department, University of Utah, Salt Lake City, UT 84112, USA \\ Saumen Datta and Steven Gottlieb \\ Department of Physics, Indiana University, Bloomington, IN 47405, USA \\ Urs M. Heller \\ CSIT, Florida State University, Tallahassee, FL 32306-4120, USA \\ Robert Sugar \\ Department of Physics, University of California, Santa Barbara, CA 93106, USA
}

(October 24, 2018)

*Present address: RIKEN-BNL Research Center, Brookhaven National Laboratory, Upton, NY $11973-5000$ 


\begin{abstract}
We present results from a lattice hadron spectrum calculation using three flavors of dynamical quarks - two light and one strange, and quenched simulations for comparison. These simulations were done using a one-loop Symanzik improved gauge action and an improved Kogut-Susskind quark action. The lattice spacings, and hence also the physical volumes, were tuned to be the same in all the runs to better expose differences due to flavor number. Lattice spacings were tuned using the static quark potential, so as a byproduct we obtain updated results for the effect of sea quarks on the static quark potential. We find indications that the full QCD meson spectrum is in better agreement with experiment than the quenched spectrum. For the $0^{++}\left(a_{0}\right)$ meson we see a coupling to two pseudoscalar mesons, or a meson decay on the lattice.
\end{abstract}

11.15Ha,12.38.Gc

Typeset using REVTEX 


\section{INTRODUCTION}

Computation of the properties of hadrons beginning from the QCD Lagrangian is a major goal of lattice gauge theory, and steady progress has been made. The computational burden of including dynamical quarks is a major obstacle in the use of lattice QCD to compute hadronic properties. As a result, many quantities are much better determined in the quenched approximation than in full QCD. One way of studying the effects of dynamical quarks is to calculate in quenched and full QCD, using the same valence quark action in both cases, and matching the lattice spacings and physical sizes of the lattices, so that any differences that are found can convincingly be ascribed to the dynamical quarks. Here we present a calculation of the hadron spectrum at a lattice spacing of about $a=0.13 \mathrm{fm}$, using quenched and full QCD lattices at the same lattice spacing. The lattice spacing was tuned by making short runs on smaller lattices, adjusting the parameters to match the lattice spacing of an initial quenched run at $10 / g^{2}=8.0$.

We use an improved Kogut-Susskind quark action which removes tree level order $a^{2}$ lattice artifacts [1]. The gauge action is a one-loop Symanzik improved action [2]. This action has been shown to reduce flavor symmetry breaking and to improve rotational symmetry of the hadron spectrum, and to give improved scaling of hadron masses as a function of lattice spacing [3].

Another important improvement of these calculations over previous generations is that we use three flavors of dynamical quarks. For quark masses larger than the strange quark mass we use three degenerate flavors, and for light quark masses less than $m_{s}$ we use two light flavors, keeping the third quark mass at about the strange quark mass. We have also done one two-flavor simulation on a matched lattice to check for effects of the dynamical strange quark. For the runs with $2+1$ dynamical flavors, we computed hadron spectra using valence quark masses equal to the sea quark masses. In the quenched run we computed hadron masses with valence quarks with the same masses, and nondegenerate propagators using a strange quark mass of $a m_{s}=0.05$. Finally, in the two dynamical flavor run we computed hadron propagators using light quark masses equal to the dynamical mass, $a m_{u, d}=0.02$ and a non-dynamical strange quark with $a m_{s}=0.05$.

Two issues that we do not completely address are extrapolation to zero lattice spacing and extrapolation to the physical light quark mass. Using matched quenched and full QCD lattices allows us to draw conclusions about the effects of dynamical quarks without explicit continuum extrapolation. Although it is in principle possible that the discretization errors in quenched and full QCD are very different, we expect that the differences in lattice spacing dependence are in fact comparable to the differences in physical quantities themselves. Since corrections to scaling are in any case quite small with our current improved action [1, 3] (see also below), we confidently expect conclusions drawn at fixed lattice spacing to survive in the continuum limit. We are beginning a series of simulations at a smaller lattice spacing which will eventually allow us to make the continuum extrapolation. A few preliminary quenched points from these finer lattice runs are included here to give an idea of the size of these effects. A complete chiral extrapolation will be more difficult. In this work, we attempt an explicit chiral extrapolation only for the shape of the static quark potential, and show other quantities as functions of the quark mass. Some quantities such as " $J$ " [4] are

only minimally sensitive to chiral extrapolation, and such quantites provide immediately 
useful tests of dynamical quark effects.

In addition to presenting hadron spectra, we update our computation of the static quark potential. For this quantity we have sufficiently accurate data that we can hazard an extrapolation to zero quark mass to produce numbers that can be compared with phenomenological potential models. Because the static potential is determined very accurately, it clearly shows differences between quenched and full QCD. In fact, one can even see the differences between two and three dynamical flavors, and a "kink" in the plots where we change from three degenerate flavors to two light and one heavy indicates a noticeable difference between two light plus one heavy and three light dynamical flavors.

In the meson sector we find differences between full and quenched QCD. A nice way of exposing these differences is the ratio $J$ proposed by Lacock and Michael [4]. We find that this quantity is in better agreement with experiment in full QCD than in the quenched approximation, as predicted in [4. This is consistent with results of the CP-PACS [5] and JLQCD [6] collaborations, who also conclude that inclusion of two flavors of dynamical quarks improves agreement of the lattice spectrum with the real world.

In the isovector $J^{P C}=0^{++}\left(a_{0}\right)$ channel we find a large difference between quenched and three-flavor results. We interpret the three flavor results as an avoided level crossing between the $0^{++}$meson and a two pseudoscalar state. In other words, we see the $a_{0}$ decay to two mesons.

We include tables of the mass fits we have chosen, so the reader can compute his or her own favorite mass ratios.

\section{SIMULATION PARAMETERS}

For our two and three flavor simulations we used the standard hybrid-molecular dynamics "R algorithm", [7] with one pseudofermion field for runs with degenerate quarks, and two pseudofermion fields for runs with different strange and up and down quark masses. In all cases, we used trajectories with unit length in the simulation time. Basic parameters of these runs are summarized in Table $\mathbb{1}$.

Two sources of systematic error in this method are the accuracy of the approximate sparse matrix solution required at each time step in the integration of the molecular dynamics equations, and the effect of the nonzero time step used in the integration. We have investigated these effects in the $2+1$ flavor simulations at $10 / g^{2}=6.8$ with two flavors of quarks at mass $a m_{u, d}=0.02$ and one flavor with $a m_{s}=0.05$ on a $16^{3} \times 48$ lattice. (These masses are approximately 0.4 times the physical strange quark mass and the physical strange quark mass, respectively). Figures 1 and 2 show the plaquette and $\bar{\psi} \psi$ as a function of the conjugate gradient residual used in the updating. Finally, to see how this effect propagates into the hadron masses, we show the Goldstone pion mass in these same runs in Fig. 3. Since the effect of this error is poorly understood, we adopted a conservative choice of $1 \times 10^{-4}$ or $5 \times 10^{-5}$ in most of our runs, and used $2 \times 10^{-5}$ for the heaviest quark mass $\left(a m_{q}=0.40\right)$ where convergence was very fast.

The effect of integration step size is better understood. We verified the expected quadratic dependence of the error on the step size in our three flavor code. The first panel in Fig. 1 shows the result of one such test, where we ran on a quite coarse lattice using the 
conventional action, comparing the old code with three degenerate flavors to the two-plusone flavor code with $m_{u, d}=m_{s}=0.02 / a$. (The "old code" uses a single pseudofermion vector, with a weight of $3 / 4$ in the fermion force, while the "two-plus-one flavor" code uses two pseudofermion vectors, one with a weight of $2 / 4$ and the other with weight $1 / 4$. In this test, both pseudofermion vectors had the same mass. In each case, the multiplication of the Gaussian random vector by $M^{\dagger}$ to produce the pseudofermion vector was done at the appropriate point in the time step to make the error in a single time step order $\epsilon^{3}$, making the accumulated error over a trajectory order $\epsilon^{2}$. [7].) It can be seen that both sets of points extrapolate to the same limit, although the size of the effect is quite different. The right hand panel of Fig. 4 shows a similar plot from one of our pre-production tuning runs with the improved gauge and quark action, at $10 / g^{2}=6.80$ and $a m_{q}=0.05$, on a $16^{3} \times 48$ lattice. This is close to the value of $10 / g^{2}$ used in the production run at this quark mass. Note that the finite step size corrections are quite sensitive to the action being used - even the sign of the effect differs between the two actions. Based on these tests and previous experience, we used a step size of no more than two-thirds of the light quark mass or 0.03, whichever was smaller, in our production runs. In the production run at $a m_{q}=0.05$ we used a step

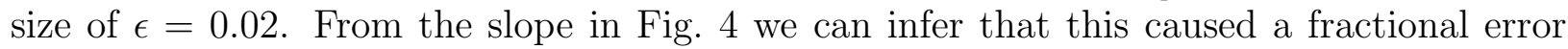
in $\langle\bar{\psi} \psi\rangle$ of about 0.004 , and a similar analysis for the plaquette suggests a fractional error of about 0.0006. We also looked at pion masses and some of the Wilson loops involved in the computation of the static quark potential, and we were unable to resolve statistically significant effects on these quantities in our tuning runs.

In our production runs with dynamical quarks we measured the potential and the spectrum at intervals of six simulation time units, and archived these lattices. The autocorrelation of the plaquette at six time units, or successive measurements, was generally about 0.1. We investigated the effect of autocorrelations on the potential and spectrum by blocking together different numbers of measurements before doing the fitting. For the potential measurements we chose to block five measurments (30 time units). For the hadron spectrum most of the particles showed no systematic effects of blocking measurements together. The exception was the pseudoscalar mesons, where blocking gave significantly better confidence levels and larger error estimates. For the pseudoscalars we chose to block together four measurements, or 24 time units.

The physical size of our lattices is $L a=20 a \approx 2.6 \mathrm{fm}$, which is similar to or larger than other recent full QCD simulations. Basic parameters of several of these calculations are summarized in Ref. [8]. 


\section{FIGURES}

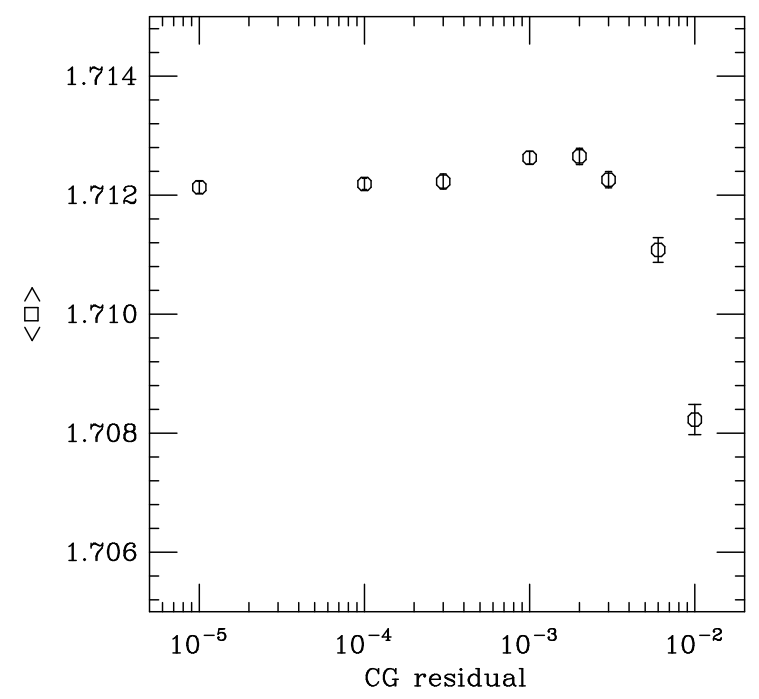

FIG. 1. The effect of the conjugate gradient error used in the updating on the plaquette in a three flavor run with quark masses $0.4 m_{s}$ and $m_{s}$.

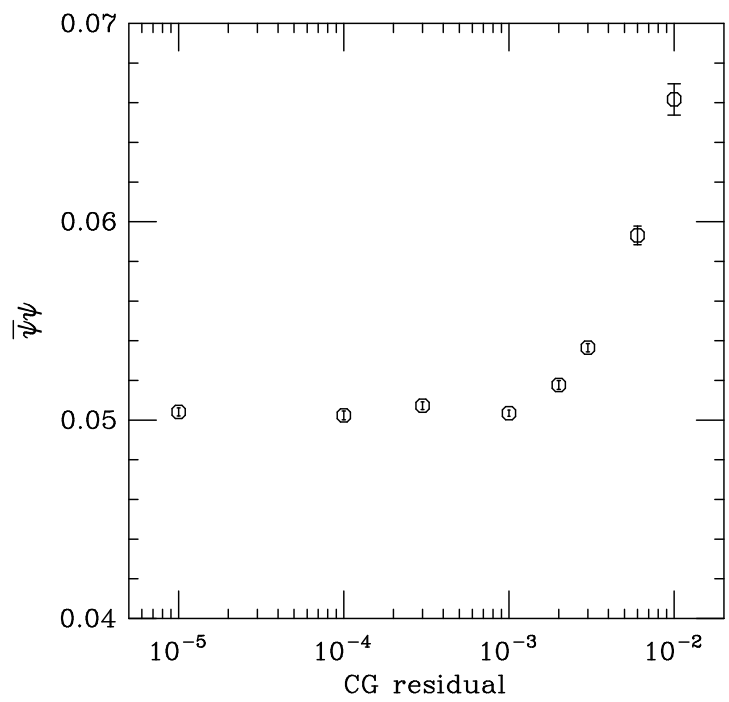

FIG. 2. The effect of the conjugate gradient error used in the updating on $\bar{\psi} \psi$ in the same three flavor run. 


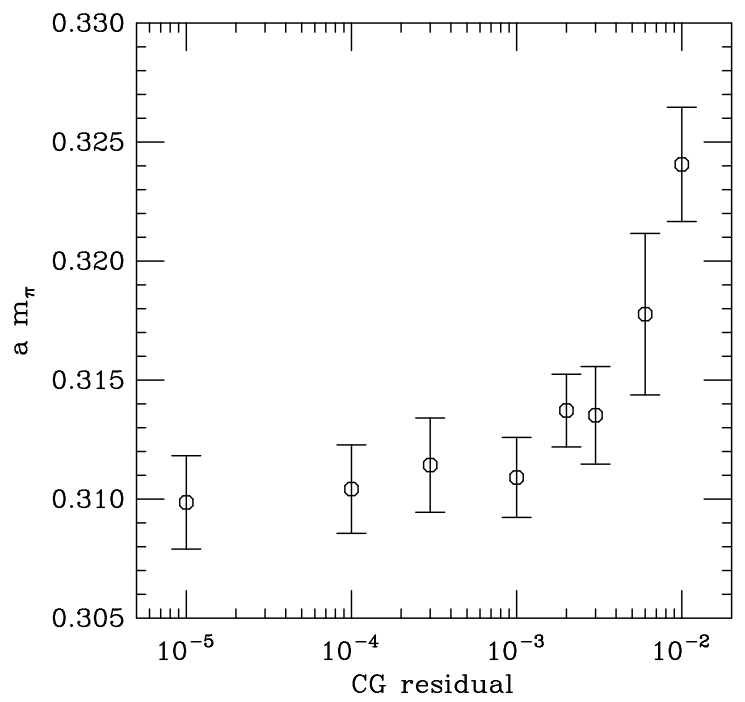

FIG. 3. The effect of the conjugate gradient error used in the updating on the Goldstone pion mass in the same three flavor run. 

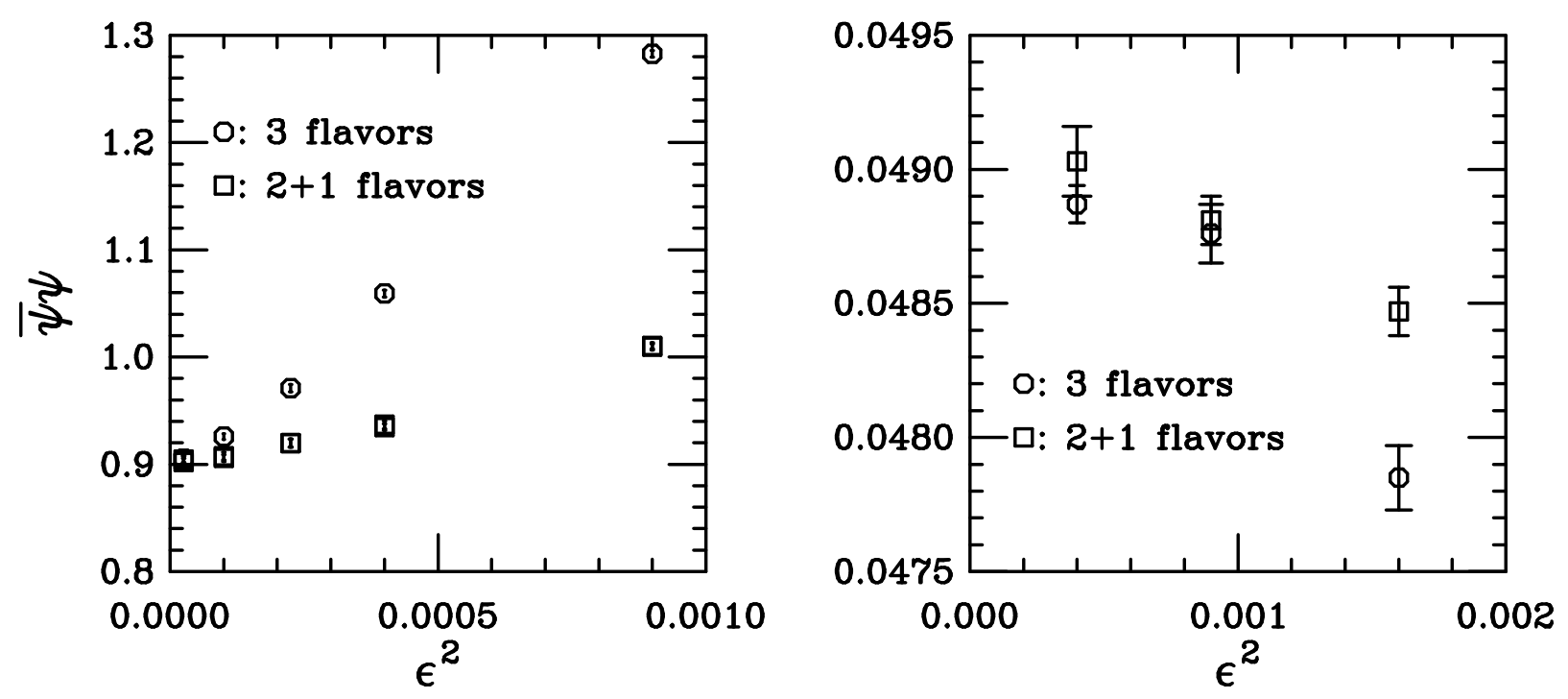

FIG. 4. $\langle\bar{\psi} \psi\rangle$ versus the squared step size. The left hand panel is an algorithm test done on a $12^{4}$ lattice using the one-plaquette gauge action and conventional quark action at $6 / g^{2}=5.10$ with three quark flavors with mass $a m_{q}=0.02$. The octagons use one pseudofermion field with a factor of $3 / 4$ in the force term, appropriate for three flavors, while the squares use the $2+1$ flavor code, with separate fermion force terms for one and two flavors, but with the same mass for both terms. The right hand panel shows $\langle\bar{\psi} \psi\rangle$ using improved gauge and quark actions at $a \approx 0.14 \mathrm{fm}$. $\left(10 / g^{2}=6.80\right.$ and $\left.a m_{u, d}=a m_{s}=0.05\right)$.

\section{LENGTH SCALES FROM THE STATIC POTENTIAL}

The static quark potential is often used to define the length scale in lattice simulations. Advantages of using the potential include ease and accuracy of its computation, and its lack of dependence on the valence quark mass. In comparing quenched and full simulations, subtleties arise because the potential does depend on the masses of the sea quarks. In Ref. [9] we demonstrated the effects of sea quarks on the potential using this improved action. Because these effects are important in our analysis of the hadron spectrum, we update and extend these results here. Our methods for computing the potential and our reasons for using $r_{1}$, a variant of the conventional $r_{0}$ [10], are described in [9]. $r_{0}$ is conventionally defined by $r_{0}^{2} F\left(r_{0}\right)=1.65$, and $r_{1}$ by $r_{1}^{2} F\left(r_{1}\right)=1.00$.

The fitting form used here is slightly more complicated than the form used in Ref. [9], with an extra term to take into account lattice artifacts at the shortest distances. Following a procedure used in Ref. [11],

$$
V(\vec{r})=C+\sigma r-\alpha / r+\lambda\left(V_{\text {free }}(\vec{r})-1 / r\right) .
$$

The last term, used for $r<2.5$, approximately compensates for remaining lattice artifacts. 
Here $V_{\text {free }}(\vec{r})$ is the potential calculated in free field theory, using the improved gauge action. Adding this term to the fits significantly improves the goodness of fit and makes the fit parameters less sensitive to the choice of distance range. For the $a \approx 0.13 \mathrm{fm}$ runs we typically find $\lambda \approx 0.3-0.4$.

In Figs. 5 and 6 we show the dimensionless quantities $r_{0} \sqrt{\sigma}$ and $r_{1} \sqrt{\sigma}$ respectively as functions of the quark mass, represented by $\left(m_{\pi} / m_{\rho}\right)^{2}$. This places the quenched approximation at $\left(m_{\pi} / m_{\rho}\right)^{2}=1$, and the chiral limit at the left side of the graph. In these plots the octagons are runs with three degenerate sea quarks, except for the rightmost point which is the quenched limit. Squares are runs with $a m_{s}=0.05$, its approximate physical value, and $a m_{u, d}<0.05$. The isolated diamond is our two flavor run. Finally, the cross at $\left(m_{\pi} / m_{\rho}\right)^{2}=1$ is the finer lattice quenched run. From the two quenched points we see that remaining lattice artifacts are small compared with the effects of the sea quarks. In particular, the central values for $r_{0} \sqrt{\sigma}$ and $r_{1} \sqrt{\sigma}$ changed by less than $1 \%$ when the lattice spacing was reduced from $0.13 \mathrm{fm}$ to $0.09 \mathrm{fm}$, a change of about $35 \%$. The kink in the plots at $\left(m_{\pi} / m_{\rho}\right)^{2} \approx 0.46\left(a m_{u, d}=0.05\right)$ shows the transition between three degenerate flavors and " $2+1$ " flavors. We can clearly see the distinction between two and three flavors, as well as the effect of using two light and one heavy flavor rather than three degenerate flavors (the "kink' at $\left.\left(m_{\pi} / m_{\rho}\right)^{2} \approx 0.46\right)$.

If we extrapolate $r_{0} \sqrt{\sigma}$ to the physical quark mass, as shown in Fig. 国, we find $r_{0} \sqrt{\sigma}=$ 1.114(4) (statistical errors only), a number which can be compared with phenomenological potential models. The two quenched points give some idea of the possible systematic error. Since the squared lattice spacing in the finer lattice is about one half that of the coarser, we might expect a shift of about twice the separation of these points in the continuum limit. Since the error bars on these points overlap, we don't know this systematic error well enough to justify such an extrapolation at this point. We expect that the effect of this systematic error is mostly an overall shift of the graphs, but the next generation of simulations should clarify this.

While using $r_{1}$ to define the length scale has the advantage that it can be done more accurately, $r_{0}$ has the advantage that it has been related to phenomenological potential models, which consistently place it around $0.5 \mathrm{fm}$ [10]. Therefore, to estimate $r_{1}$ in physical units, we plot $r_{0} / r_{1}$ in Fig. 7. Extrapolating linearly in $\left(m_{\pi} / m_{\rho}\right)^{2}$ to the physical value gives $r_{0} / r_{1}=1.449(5)$ (statistical error only), or with $r_{0}=0.5 \mathrm{fm}, r_{1}=0.35 \mathrm{fm}$. 


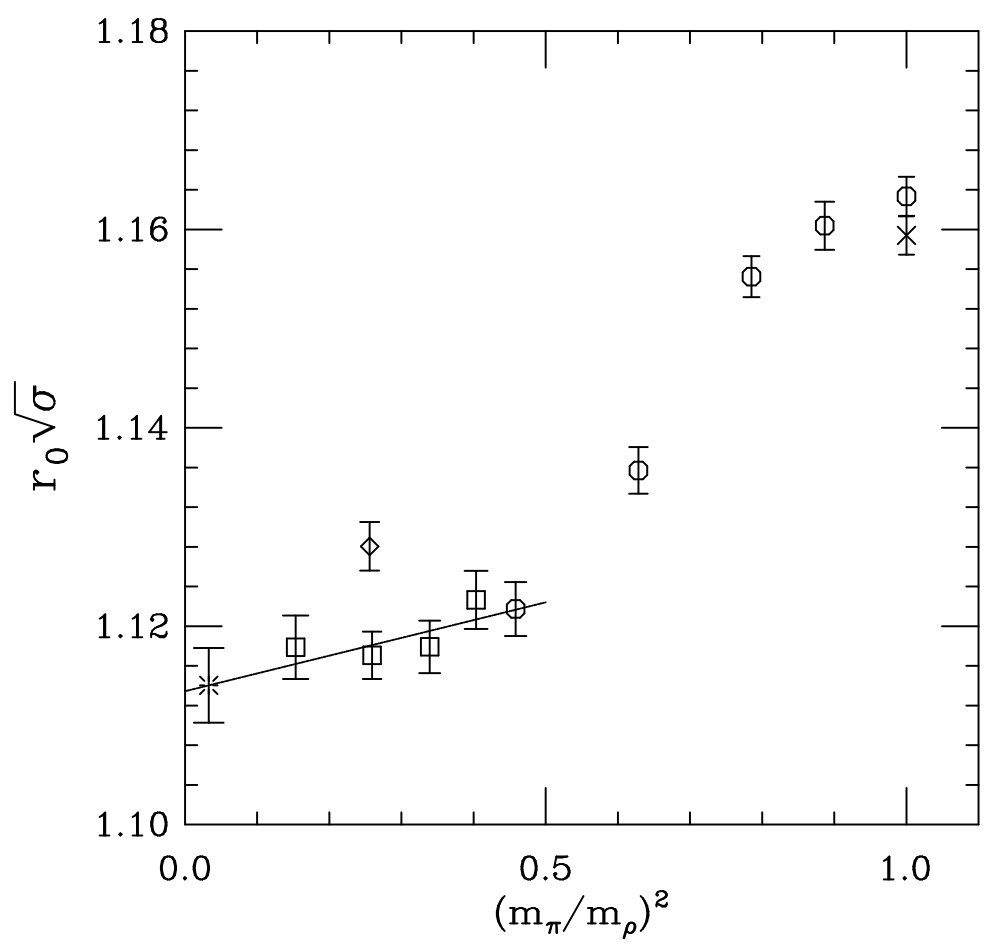

FIG. 5. Effects of dynamical quarks on the shape of the potential. Here we plot $r_{0} \sqrt{\sigma}$ as a function of the quark mass. The two quenched points are at the far right, with the octagon coming from the $10 / g^{2}=8.0$ run and the cross from the $10 / g^{2}=8.4$ run, which has a lattice spacing of about $0.09 \mathrm{fm}$. The remaining octagons are full QCD runs with three degenerate flavors, and the squares are full QCD runs with two light flavors and one heavy. The diamond is the two flavor run, and the burst at the left is a linear extrapolation of the $2+1$ results to the physical value of $\left(m_{\pi} / m_{\rho}\right)^{2}$. 


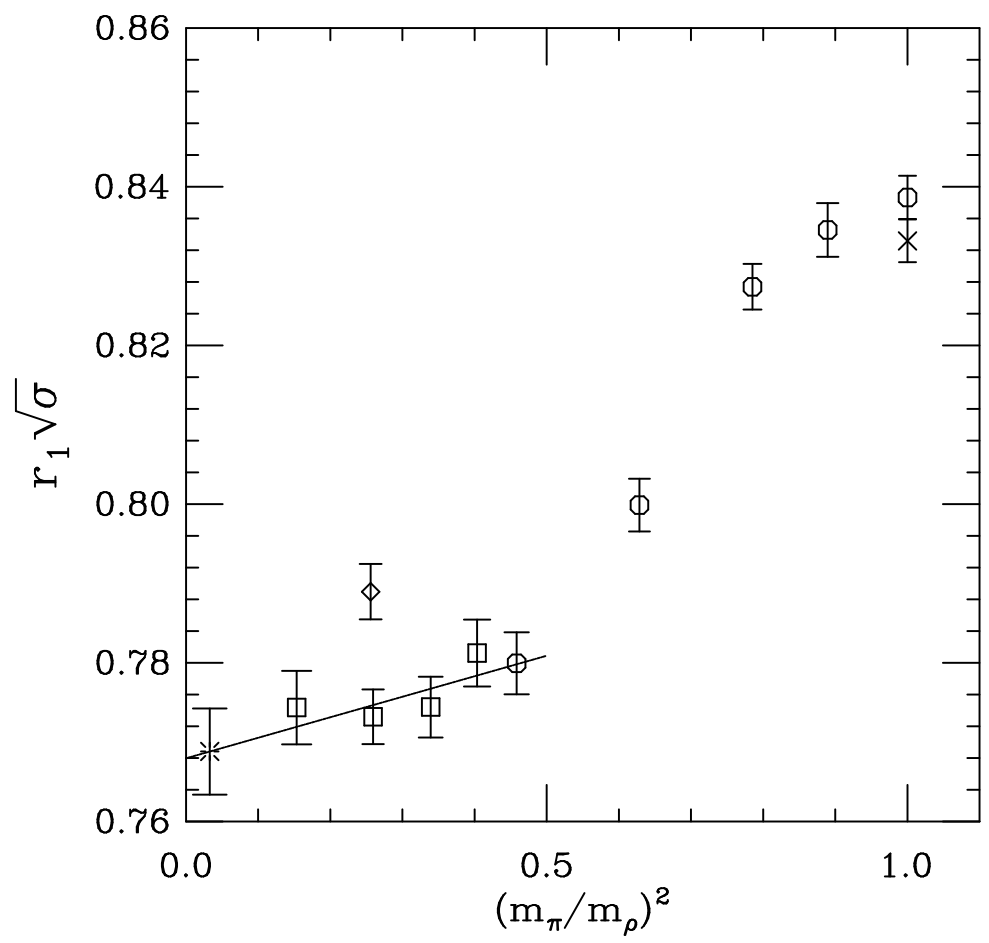

FIG. 6. The same as Fig. 5, except we plot $r_{1} \sqrt{\sigma}$. Physically, the difference is that this quantity is sensitive to shorter distances than $r_{0} \sqrt{\sigma}$.

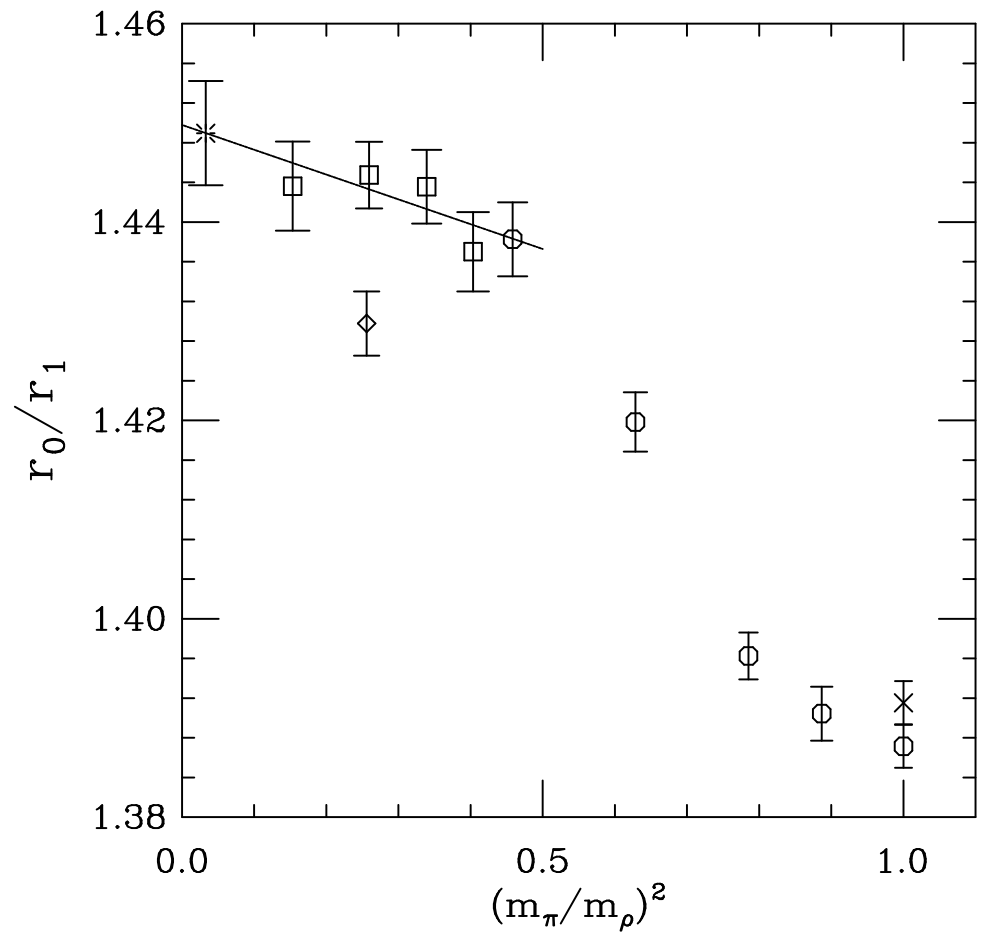

FIG. 7. The ratio $r_{0} / r_{1}$, and a chiral extrapolation. The symbols are the same as in Fig. 5 . 


\section{PROCEDURES FOR DETERMINING MASSES}

All of our hadron propagators used wall sources and local sink operators. Several different wall sources were used. For the "pointlike" hadrons, for which all the quarks can be on a single corner of the hypercube, a "corner wall" source gave the best results. This source is simply a 1 on each $(0,0,0)$ corner of the $2^{3}$ cubes on the chosen time slice. However, to isolate the decuplet baryons a non-pointlike source is essential, and we used "even" and "odd" wall sources, where 1 or $(-1)^{x+y+z}$ is placed on each site, respectively. This set of sources was developed in Ref. [12]. For the nonlocal pseudoscalar and vector mesons we used two wall sources made from empirically determined linear combinations of the nonlocal pion operators. Finally, for the nonzero momentum mesons we used a quark source with 1 on each site, and an antiquark source with $e^{i \vec{k} \cdot \vec{x}}$ on each site. All of the configurations were gauge fixed to the Coulomb gauge before computing the propagators.

In most cases we computed propagators from four source times evenly spread through the lattice (only one source slice was "turned on" at a time). For the corner source we used eight source time slices for the light quark particles with $a m_{q} \leq 0.04$, and we used eight source time slices for the "even" and "odd" source baryon propagators. (The $\Delta$ propagator is very noisy, and propagators computed from source times separated by $8 \times 0.13 \mathrm{fm}$ were basically independent.)

For Kogut-Susskind quarks the meson propagators have the generic form

$$
\mathcal{H}(t)=\sum_{i} A_{i}\left(e^{-m_{i} t}+e^{-m_{i}\left(N_{t}-t\right)}\right)+\sum_{i} A_{i}^{\prime}(-1)^{t}\left(e^{-m_{i}^{\prime} t}+e^{-m_{i}^{\prime}\left(N_{t}-t\right)}\right) .
$$

Here the oscillating terms correspond to particles with opposite parity from the ordinary exponential terms. Baryon propagators are similar, but have antiperiodic boundary conditions and the "backwards" terms include an extra factor of $(-1)^{t}$. In most cases only one mass with each parity is included in the fits, but for half of the pseudoscalar meson operators the opposite parity terms are not present, and for the P-wave mesons we found it necessary to keep two simple exponentials. The quantum numbers for the various operators are tabulated in Ref. [13].

Hadron masses were determined from fits to propagators, using the full covariance matrix to estimate errors. The maximum time distance used in the fits was chosen to include points with fractional error less than 0.3. Because of the oscillating components in the staggered quark propagators, it sometimes happens that the fractional error exceeds the threshold at one distance but is smaller at larger distances, so the complete criterion for the maximum distance included is the largest distance such that the fractional error on each of the next two points exceeds 0.3. Since the points at largest distance contribute little information, the exact large distance cutoff is not critical. To choose the minimum distance included, we first went through the fits and chose a minimum distance for each hadron in each dataset, choosing a distance where the confidence level was reasonable and where the mass appeared to reach a plateau. As expected, some propagators had larger(smaller) fluctuations than other similar propagators, resulting in the choice of a larger(smaller) minimum distance. For the particles of greatest interest, to reduce this effect we then "smoothed" these minimum distances, requiring that the minimum distances be smooth functions of quark mass and be the same for the quenched and dynamical runs. The resulting minimum distances are 
strongly dependent on the quark masses, with smaller minimum distances for smaller masses. Most of this is due to the larger statistical errors at smaller quark mass, which result in the excited state contributions disappearing into the noise at shorter distance. However, it is in part physical, since splittings between the ground and excited states are larger for smaller quark masses. Table 11 shows the minimum distances that we chose, and the number of particles with each parity.

\section{RESULTS}

\section{A. Pseudoscalar mesons}

We calculated propagators for all eight flavor combinations of the staggered quark pseudoscalar mesons. These masses obey the "partial flavor symmetry restoration" predicted by Lee and Sharpe [14] to very good accuracy. Specifically, Ref. [14] predicts that the leading order flavor symmetry breaking effects, which are order $a^{2}$, leave degeneracies between pairs of pseudoscalar mesons for which $\gamma_{0}$ is replaced by $\gamma_{i}$ in the flavor structure. For example, the local non-Goldstone pion, $\gamma_{0} \gamma_{5} \otimes \gamma_{0} \gamma_{5}$ in the "spin $\otimes$ flavor" notation, is degenerate with the distance one pion, $\gamma_{5} \otimes \gamma_{i} \gamma_{5}$, to this order. Moreover, all of the squared pion masses should depend linearly on the quark mass with the same slope to lowest order. Figure 8 shows this behavior for the quenched pion masses for $a m_{u, d} \leq 0.05$. The results for the full QCD runs are similar. Since the local $\gamma_{5} \otimes \gamma_{5}$ pseudoscalar has the correct chiral behavior (and the best scaling behavior), we will use this pseudoscalar in the rest of the analysis unless we specifically indicate otherwise. Results for the full QCD runs are similar, but the flavor symmetry breaking is somewhat larger.

In Fig. 8 the relation between the squared pseudoscalar mass and the quark mass is clearly nearly linear. The deviations from linearity and the effect of the dynamical quarks can be exposed by plotting the squared pseudoscalar masses divided by the quark mass, in Fig. 9. This is essentially $\langle\psi \bar{\psi}\rangle r_{1} / f_{\pi}^{2}$ with an (unknown) renormalization factor. This plot contains pseudoscalar mesons with both light and strange valence quarks (pions, kaons and "unmixed $s \bar{s}$ 's"). There is clearly a systematic difference between quenched and full QCD. This difference increases with decreasing quark mass, and the two-flavor point falls in between the quenched and three-flavor points. The bursts among the quenched points are from the $10 / g^{2}=8.4, a \approx 0.09 \mathrm{fm}$ run, showing gratifying agreement with the $a \approx 0.13$ $\mathrm{fm}$ points. Unfortunately, a coarser three flavor lattice, $a \approx 0.2 \mathrm{fm}$, shows a large effect, so we would not want to use much coarser lattices in studying this effect. We do note that we expect scaling violations to be similar for the quenched and dynamical theories, so it is an advantage to have runs with matched lattice spacings. The deviations from linearity of $m_{\pi}^{2}$ are similar in quenched and full QCD. The upturn for larger quark masses signals the beginning of the transition to the heavy quark regime, where $m_{\pi}^{2} \approx m_{q}^{2}$. We do not fully understand the shape of this plot for small quark mass. There are several ways to interpret the difference between quenched and dynamical results. One could say that $\langle\bar{\psi} \psi\rangle / f_{\pi}^{2}$ is too small in the quenched approximation, or one could say that the quark mass at which a desired $m_{\pi} / m_{\rho}$ is reached is larger in the quenched approximation than in full QCD. This second interpretation is consistent with CP-PACS results on the quark masses, in which they find that the quark mass needed to reach a given value of $m_{\pi} / m_{\rho}$ is smaller in two 
flavor QCD than in quenched QCD [15]. (Indeed, one could even use this quantity as a length scale, and conclude that $r_{1}$ is different in quenched and full QCD.)

The largest part of the error bars in Fig. 9 come from the uncertainty in $r_{1}$. However, this uncertainty is common to all of the points coming from the same set of lattices. In particular, all the $a \approx 0.13 \mathrm{fm}$ quenched points are correlated in this respect, as are the three two-flavor points. If we are interested in the dependence of the pseudoscalar mass on the quark mass on a fixed lattice, we may want to consider only the error from the determination of the meson mass in units of $a$. The left hand panel in Fig. 10 shows $m_{\pi}^{2} r_{1}^{2} /\left(r_{1}\left(m_{1}+m_{2}\right)\right)$ for the quenched calculation, including only the error from $a m_{P S}$ and showing only the reasonably light mass points. In this panel the octagons are "pions", with $m_{1}=m_{2}=a m_{u, d}$, and the bursts are "kaons", with $a m_{2}$ fixed at 0.05 , which is approximately the physical value of the strange quark mass. We see that this quantity is dependent only on the sum of the quark masses to very good accuracy. The center panel contains the same plot for the three-flavor runs, where now $r_{1}$ is determined independently in each run. In this panel the octagons are runs with $a m_{u, d}=a m_{s} \geq 0.05$, the squares the run with $a m_{u, d}=0.04$, the diamonds from $a m_{u, d}=0.03$, the crosses from $a m_{u, d}=0.02$ and the bursts from $a m_{u, d}=0.01$. The three symbols for each of the runs with $a m_{u, d}<0.05$ correspond to the "pion", with both valence quarks light, the "kaon", with one light and one strange valence quark, and an "unmixed $s \vec{s}$ ", with two valence quarks of mass approximately equal to that of the strange quark, but no $q \bar{q}$ annihilation. This graph is far from smooth, but most of the scatter comes from the fact that each set of dynamical quark masses has an independent uncertainty in $r_{1}$. Note that each dynamical run (for example, the three bursts) shows qualitatively the same behavior as the quenched case, with the light-light pseudoscalar tending to a larger value. An interesting question is how the sea quark mass affects the pseudoscalar mass. This can be investigated by looking at the points at $a\left(m_{1}+m_{2}\right)=0.1$, which are the "unmixed $s \bar{s}$ " points with both valence quark masses equal to $0.05 / a$. In the right hand panel of Fig. 10 we plot these points as a function of the light quark mass. There is a noticeable effect, with smaller light quark mass producing larger $s \bar{s}$ mass. The direction of this effect is consistent with the smaller pseudoscalar masses in the two flavor and quenched calculations seen in Fig. 9 .

The selected pseudoscalar meson mass fits in units of the lattice spacing are tabulated in table III. In addition to the "pions", the table also contains fits with one quark at about the strange quark mass and one lighter quark, or "kaons". For the two and three flavor runs we also tabulate "unmixed $s \bar{s}$ mesons", with two valence quarks with $a m_{v}=0.05$.

We have attempted to fit the results in Fig. 9 to the forms predicted by chiral perturbation theory. In the quenched case, the behavior of $m_{\pi}^{2}$ as a function of quark mass is derived in Refs. [16, 17]. We use eqn. (9) in [16], with the parameter $\alpha$, which is believed to be small, set equal to zero, and the analytic correction term added to the chiral log. For pions, then one has

$$
\frac{m_{\pi}^{2}}{m}=C\left(1-\delta \log \left(\frac{C m}{\Lambda^{2}}\right)+K m\right)
$$

where $m_{1}=m_{2} \equiv m, C$ and $K$ are constants, and the chiral scale $\Lambda$ may be taken as the $\eta$ mass. As shown in Fig. 9, the fit to the $a=.13 \mathrm{fm}$ quenched data is good. It gives $\delta=0.061(3)$ (statistical error only), which is on the low side but compatible with the range 
reported by CP-PACS [18] and is in excellent agreement with the result of Bardeen et al. [19], $\delta=0.065(13)$.

Unfortunately, our attempts to fit the 3-flavor pion data in Fig. 9 to the corresponding full QCD chiral form [20] have been unsuccessful to date. We can fit the 5 lowest-mass pions with reasonable confidence level, but the coefficients of the analytic terms are unreasonably large, and the fit misses the next lightest pion by a wide margin. If we try to fit the 6 lowest-mass pions, the fit has terrible confidence level. Finally, good fits can be obtained by introducing, as an additional free parameter, an overall coefficient in front of the chiral logs. However, the value of that coefficient in the fit is much smaller than its predicted [20] value. We are continuing to study this puzzling situation. Our current running at smaller lattice spacing may provide additional insight here. 


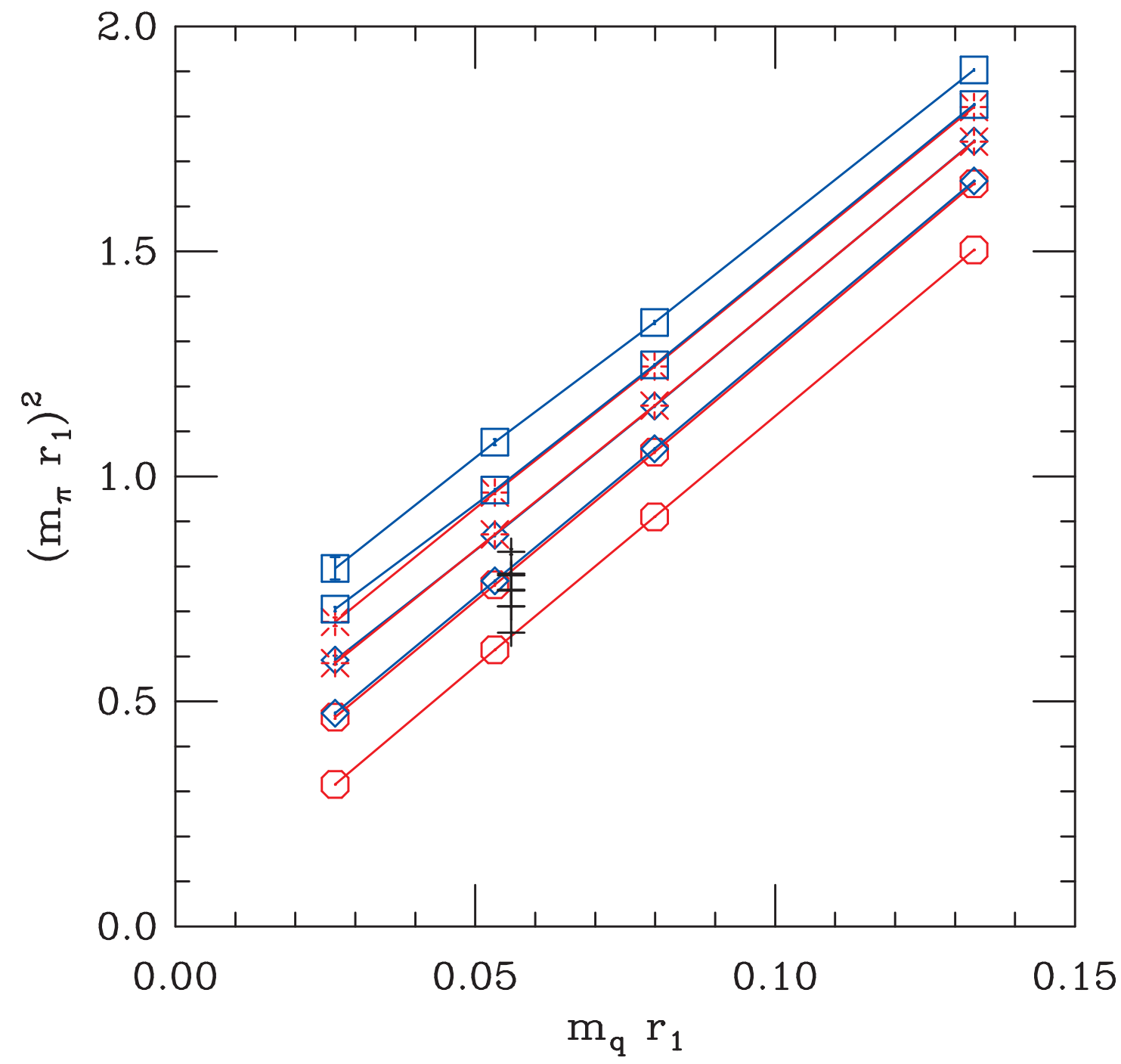

FIG. 8. Squared pseudoscalar meson masses versus quark masses. These results are from the quenched runs. The octagons are the local pions $\left(\gamma_{5} \otimes \gamma_{5}\right.$ and $\left.\gamma_{0} \gamma_{5} \otimes \gamma_{0} \gamma_{5}\right)$, the diamonds the distance one pions, the bursts the distance two pions, and the squares the distance three pions. The degeneracies predicted in Ref. [14] are clearly visible. The lines are not fits; they simply connect the points. The column of pluses is from the quenched $a \approx 0.09 \mathrm{fm}$ run, showing the expected improvement in flavor symmetry with decreasing lattice spacing. Note that the Goldstone pion changes very little when the lattice spacing is decreased - the non-Goldstone pions come down to join it. 


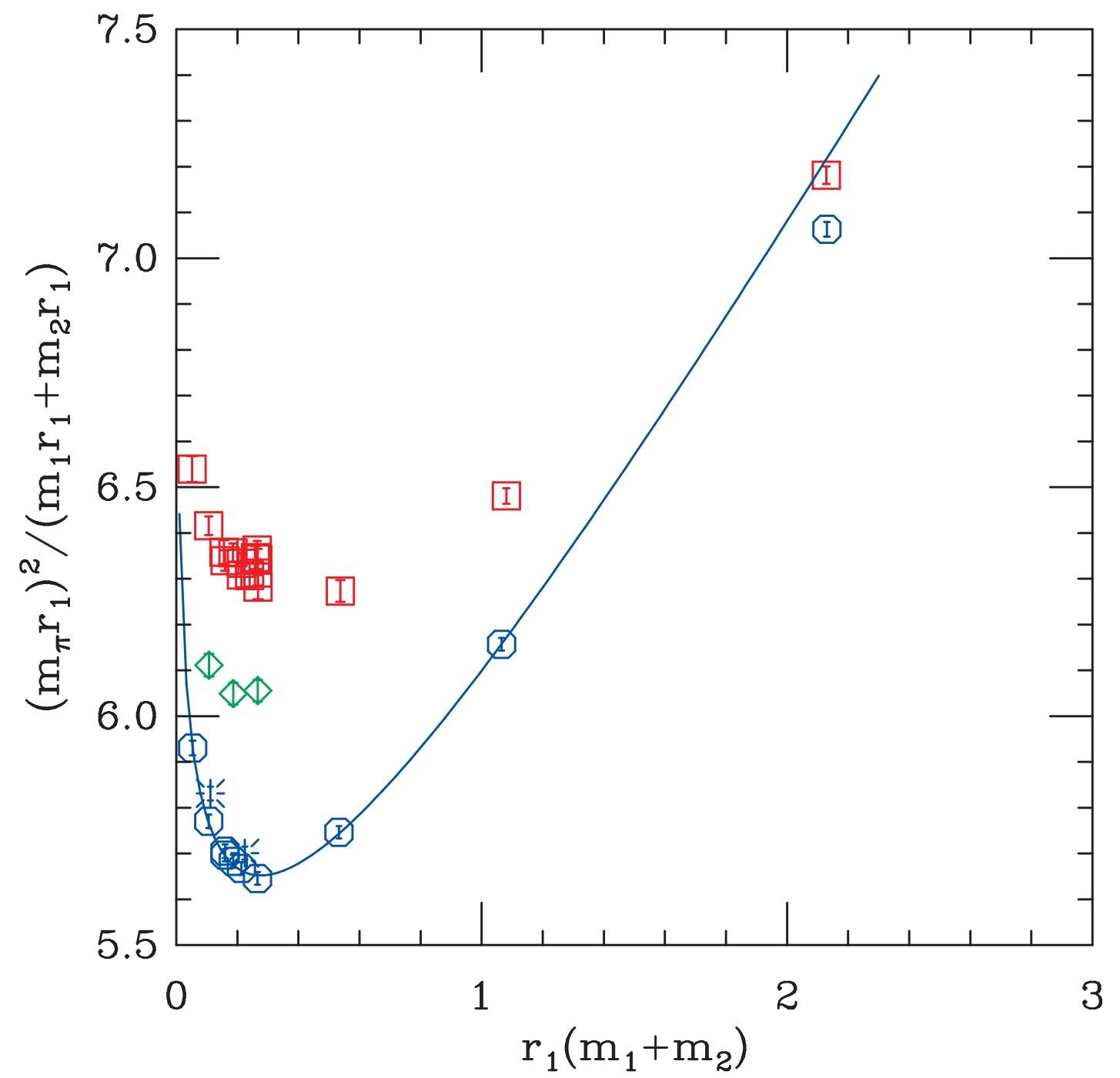

FIG. 9. The squared pseudoscalar mass divided by the quark mass in units of $r_{1}$. The octagons are the quenched $a \approx 0.13 \mathrm{fm}$ run, and the squares the three flavor $a \approx 0.13 \mathrm{fm}$ runs. The diamonds are the single two-flavor run. The bursts are from the quenched $a \approx 0.09 \mathrm{fm}$ run. The fit is to the octagons (pions only) with $r_{1}\left(m_{1}+m_{2}\right)<1.4$, using the form in eq. (阴). 

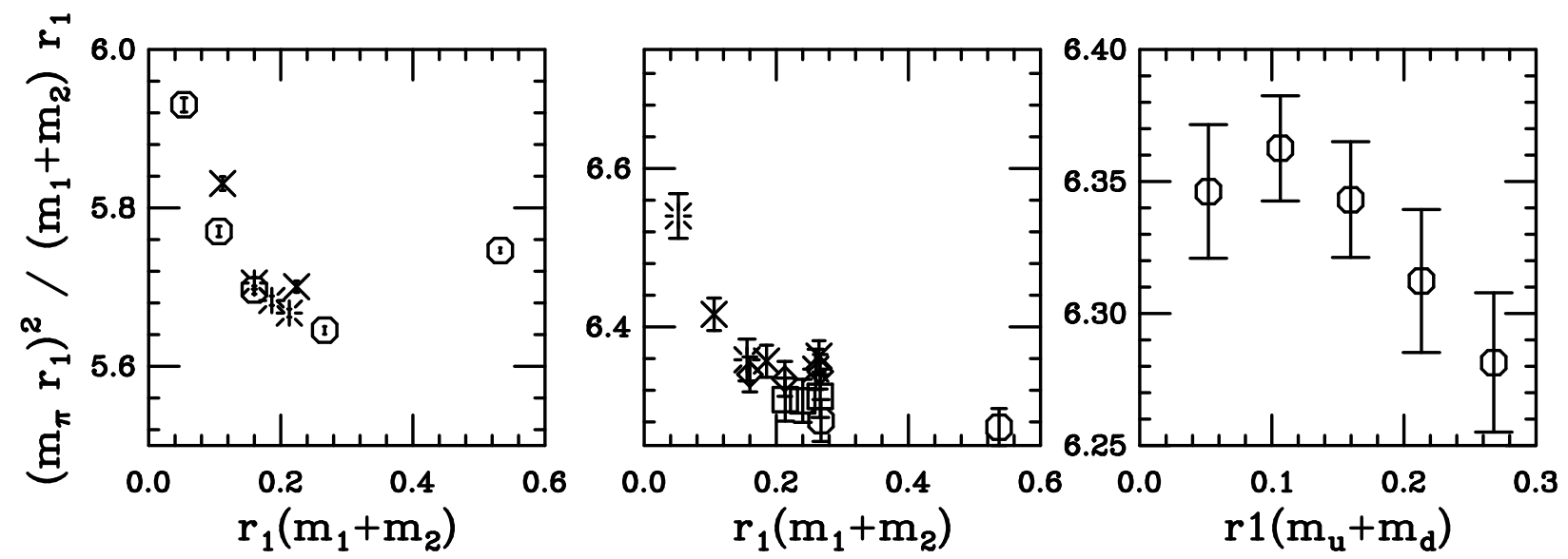

FIG. 10. Details of $\left(m_{P S} r_{1}\right)^{2} /\left(r 1\left(m_{1}+m_{2}\right)\right)$. The three panels are the quenched results, the dynamical results, and the three flavor points with two strange valence quarks.

\section{B. Vector mesons}

We calculated propagators for the two local vector mesons, $\gamma_{i} \otimes \gamma_{i}$ (VT) and $\gamma_{0} \gamma_{i} \otimes \gamma_{0} \gamma_{i}$ $(\mathrm{PV})$, and two distance one vector mesons, $\gamma_{i} \otimes \mathbf{1}$ and $\gamma_{0} \gamma_{i} \otimes \gamma_{0}$. Any flavor symmetry breaking among these mesons is smaller than the statistical errors, so we simply quote results for the local $\gamma_{i} \otimes \gamma_{i}$, or "VT" mesons. Table IV contains these masses in units of the lattice spacing.

In Figure 11 we plot the vector meson mass in units of $r_{1}$ versus the squared pion/rho mass ratio. In this plot there is a clear difference between the quenched and dynamical masses, with the full QCD vector mesons lying lower. However, the size of this effect depends on the length standard chosen, as illustrated in Fig. 12, where the same quantity is plotted in units of the string tension. Of course, the difference between these two plots simply arises from the difference in $r_{1} \sqrt{\sigma}$ plotted in Fig. 6 . 


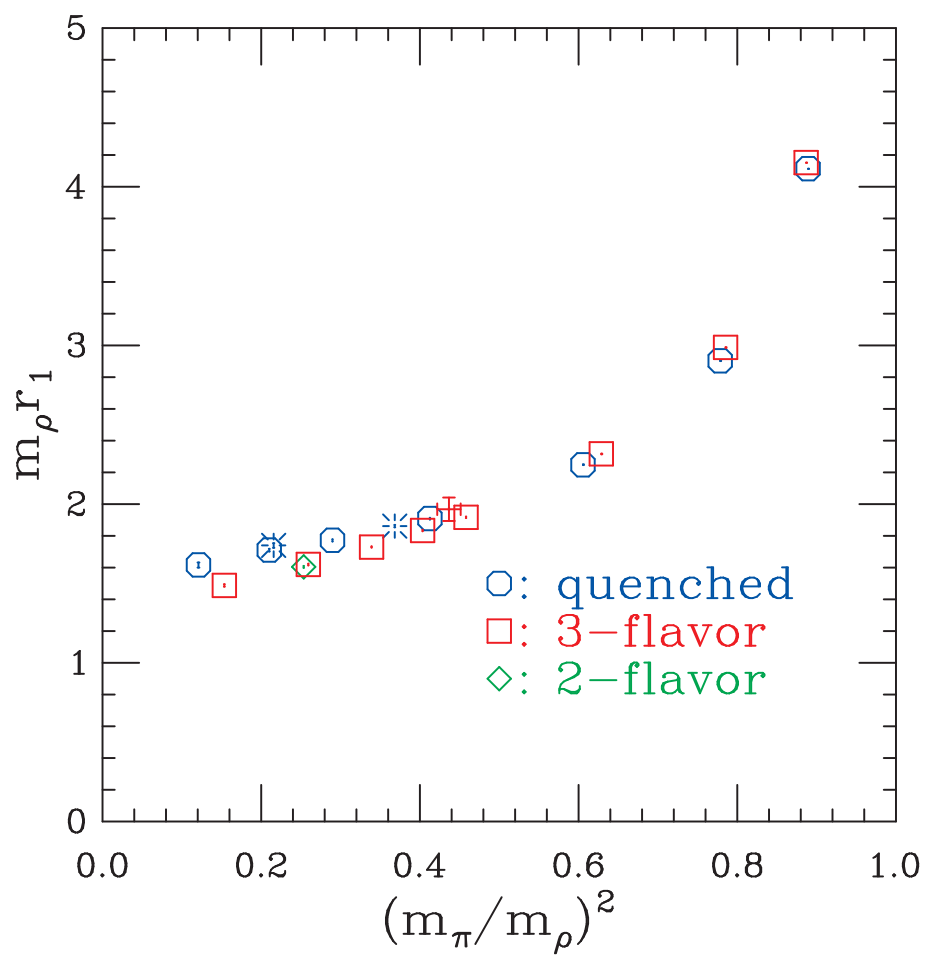

FIG. 11. Vector meson masses in units of $r_{1}$. The octagons are quenched results, the diamond a two-flavor result, and the squares three-flavor. The bursts are quenched $a \approx 0.09 \mathrm{fm}$ points, and the fancy plus an $a \approx 0.2 \mathrm{fm}$ three flavor point.

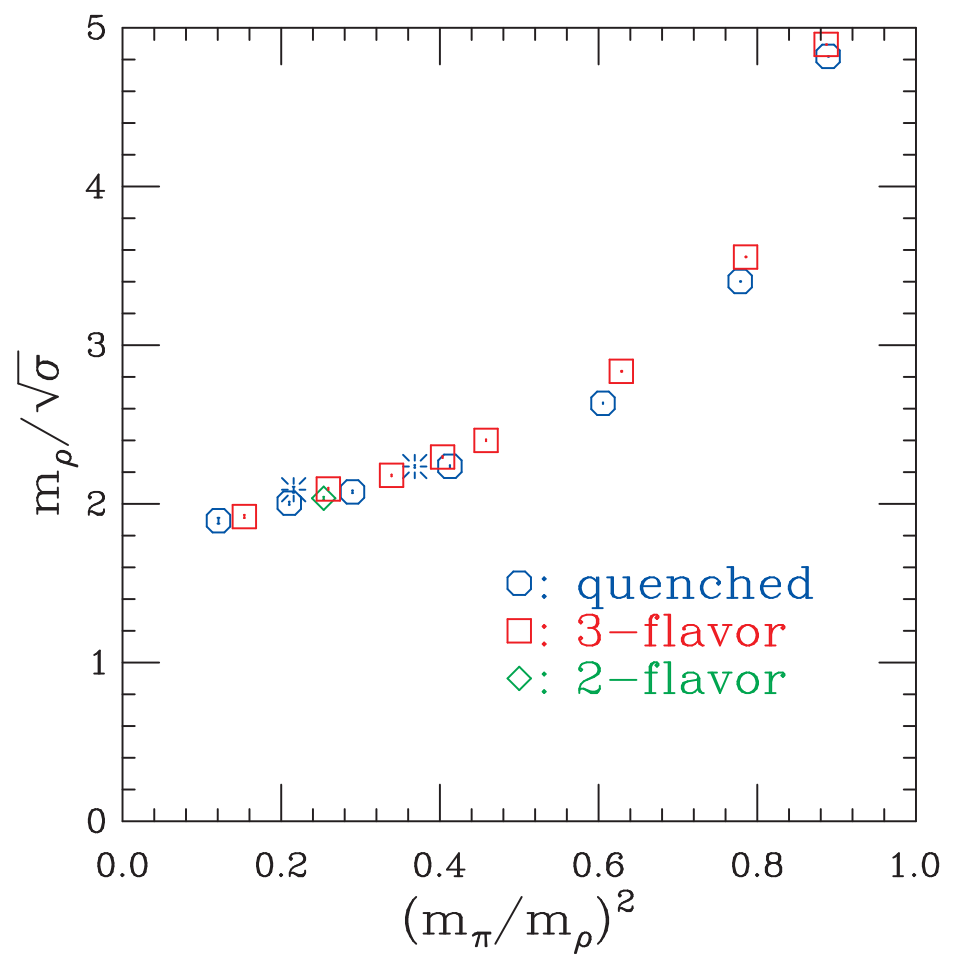

FIG. 12. The same as Fig. 11, except in units of the string tension. 
In our calculations we used a mass of $a m_{s}=0.05$ for the strange quark mass. With the meson spectrum in hand, we can now go back and ask whether this choice was exactly correct. There are several quantities that we could choose to define the strange quark mass, and, especially in the quenched calculation, they will in general give different results. Perhaps the most accessible quantity is the ratio of the $s \bar{s}$ pseudoscalar to vector meson mass. (Our "s $s \bar{s}$ pseudoscalar" does not include $q \bar{q}$ annihilation diagrams, so it is not the $\eta$ or $\eta^{\prime}$.) We therefore tune this quantity using $m_{s \bar{s}}=\sqrt{2 m_{K}^{2}-m_{\pi}^{2}}=686 \mathrm{MeV}$ and $m_{\phi}=1020 \mathrm{MeV}$, or $m_{P S} / m_{V}=0.673$.

In the quenched spectrum with $a m_{q}=0.05$ we have $m_{P S} / m_{V}=0.643$, indicating that $a m_{q}=0.05$ is smaller than the desired strange quark mass. To compute the mass at which $m_{P S} / m_{V}=0.673$, we can do a linear fit to the vector meson mass as a function of quark mass, and combine that with a squared pseudoscalar mass proportional to the quark mass to conclude that the quenched strange quark mass defined by $m_{P S} / m_{V}$ at this lattice spacing is $a m_{s}=0.058$. In contrast, for the dynamical runs $a m_{s}=0.05$ is a fairly good estimate of the strange quark mass, with $m_{P S} / m_{V}$ taking values between 0.670(2) and 0.687(1).

Differences between the quenched meson spectrum and the real world have been observed by the UKQCD collaboration [4], and improvements of the spectrum when dynamical quarks are included have been reported by the CPPACS [5] and JLQCD [6] collaborations. In particular, the UKQCD collaboration studied the quantity

$$
J=m_{K^{*}} \frac{\partial m_{V}}{\partial m_{P S}^{2}},
$$

where $m_{V}$ and $m_{P S}$ are the vector and pseudoscalar meson masses. This quantity has the advantage of being relatively insensitive to the quark masses, so that accurate tuning of the strange quark mass or extrapolation of the masses to the chiral limit is not essential. Of course, to compare to experiment the derivative in this expression must be replaced by a ratio of mass differences, and we choose

$$
J=m_{K^{*}} \frac{\left(m_{\phi}-m_{\rho}\right)}{2\left(m_{K}^{2}-m_{\pi}^{2}\right)} .
$$

Here $m_{\rho}$ is the mass of the vector meson including two light quarks, etc. We choose the $m_{\phi}-m_{\rho}$ mass difference because the statistical error in $J$ is dominated by the error in the vector meson masses, and the larger difference in $m_{\phi}-m_{\rho}$ relative to, say, $m_{K^{*}}-m_{\rho}$ leads to smaller statistical errors on $J$. Because all of the masses in Eq. 5 are correlated, we use a jackknife analysis to compute the error on $J$. Figure 13 shows the results for $J$ in quenched and three flavor QCD. Following UKQCD, we plot this versus $m_{K^{*}} / m_{K}$, for which the real world value is 1.8. The burst is the real world value of this definition of $J$ (0.49), and the cross is the value of $J$ found in the UKQCD quenched simulations. We see a clear effect of the sea quarks on this quantity. Indeed, any reasonable extrapolation of our data in $m_{K^{*}} / m_{K}$ would pass near the real world point. Figure 13 also contains one point with two dynamical flavors. This point falls near the three flavor points, indicating that the dynamical strange quark is less important than the two light quarks. Although our quenched results are somewhat higher than the UKQCD value, they are significantly below the experimental value. The fact that the quenched points in this plot are to the right of the full QCD points is largely due to the fact that the mass of $a m_{q}=0.05$ used for the 
quenched strange quark was too small, as discussed above. If we use the observed slopes of the quenched vector meson masses and squared pseudoscalar masses as functions of quark mass to adjust these points to a quenched strange quark mass of $a m_{s}=0.058$, the main effect is to shift the quenched points to the left. In particular, the rightmost quenched point moves to $m_{K^{*}} / m_{K}=1.78$, but moves up by only 0.004 .

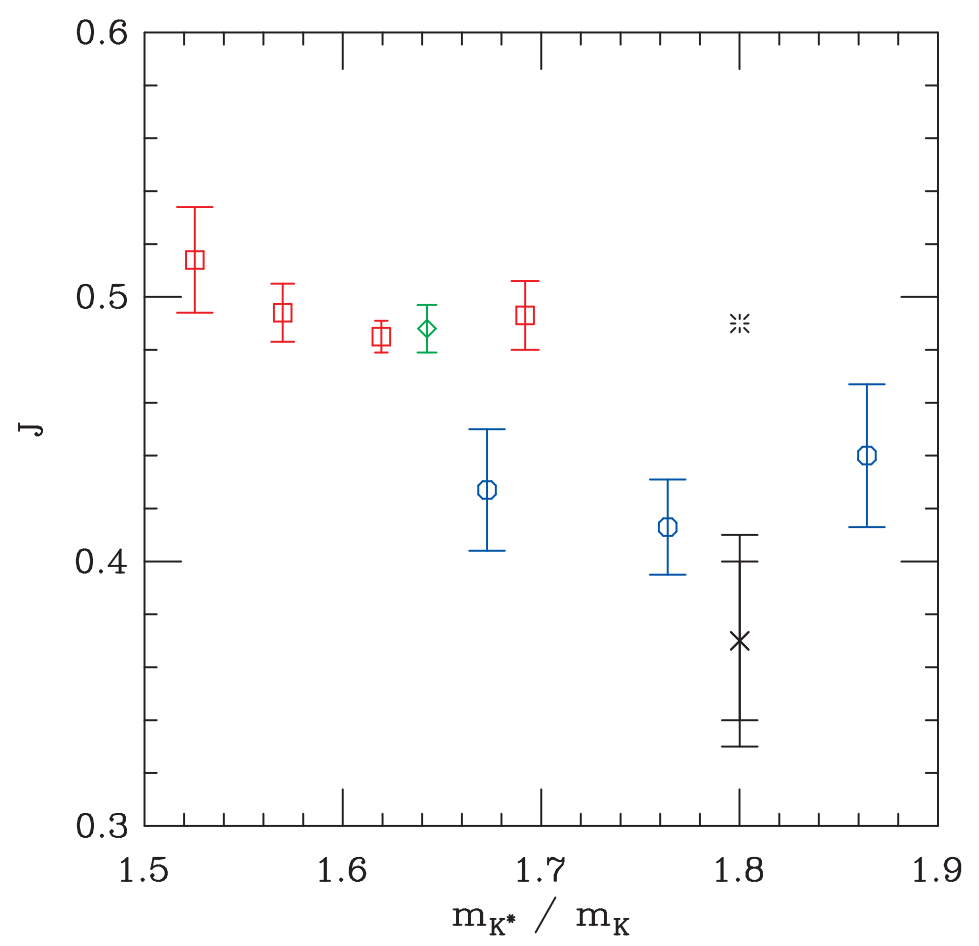

FIG. 13. The mass ratio " $J$ " in the quenched and full QCD calculations. Squares are the three flavor results, and octagons are the quenched results. The diamond is the two flavor run, using a non-dynamical strange quark with mass $a m_{q}=0.05$. The burst is the real world value, and the cross is the UKQCD quenched value. The smaller error bar on the cross is the statistical error, and the larger the quoted systematic error.

\section{P-wave mesons}

Mesons with quantum numbers $J^{P C}=0^{++}, 1^{++}$and $1^{+-}$, which are P-wave mesons in the nonrelativistic limit, are found as the oscillating "parity partners" of the $\pi$ and $\rho$ propagators with Kogut-Susskind quarks. Fitting these particles is difficult because of their larger mass. In order to get good fits, we need to allow two particles in the non-oscillating components, since we typically find that the excited " $\pi$ " or " $\rho$ " state is comparable in mass to the lowest oscillating (P-wave) state. Thus we find large errors, and must use small minimum distances to get good fits. In addition, plateaus in the effective mass are short. Figure 14 illustrates an example of a difficult but crucial case. This figure shows fits to the scalar $\left(a_{0}\right)$ mass as a function of the minimum distance included in the fit. With these caveats, selected fits for the P-wave mesons are shown in Fig. [15 and Tables $\nabla, \nabla]$ and VII. This figure and these tables contain several interesting features. For the $a_{1}$ and $b_{1}$ the 
full QCD runs give consistently better fits than the quenched run, and smaller masses for the lighter quarks. The $b_{1}$ is consistently slightly heavier than the $a_{1}$, although with the difficulties in extracting these particles we would not want to make too much of this. The diamond at the left in Fig. 15 is the experimental value for the $a_{1}$ and $b_{1}$ masses.

The scalar channel, " $a_{0}$ ", is clearly very different in the quenched and full QCD runs. For large quark masses there is no visible difference, but as the quark mass is decreased the full QCD $0^{++}$mass drops below all the other masses. For all but the lowest quark mass, the quenched $0^{++}$is close to the other P-wave meson masses. We ascribe the behavior of the full QCD mass to the decay of the $a_{0}$ into $\pi+\eta$. (Bose symmetry plus isospin forbids decay into two pions.) Figure 16 illustrates this interpretation. In the figure we plot the quenched and full $0^{++}$masses versus quark mass. The straight line in the graph is a fit to the quenched mass for the heavier quarks, and represents the mass of a $q \bar{q}$ state. The curved line with the kink at $a m_{q}=0.05$ represents the mass of $\pi+\eta$. For $a m_{q} \geq 0.05$ we used three degenerate quark flavors, so the $\eta$ and $\pi$ are degenerate and this line is simply twice the pion mass. For $a m_{q}<0.05$ we don't have direct information on the $\eta$ mass, so we use the Gell-man-Okubo formula written in terms of an "unmixed $s \vec{s}$ " mass (just our pseudoscalar mass at $\left.a m_{q}=0.05\right)$.

$$
m_{\eta}^{2}=\left(m_{\pi}^{2}+2 m_{s \bar{s}}^{2}\right) / 3
$$

In the quenched case the $a_{0}$ mesons can couple to two-meson states through a "hairpin diagram" on one of the meson lines. Such diagrams, like Fig. 1(b) in Ref. [21], can behave like powers of $t$ times $e^{-2 m_{\pi} t}$ and therefore masquerade as a light $a_{0}$ when $2 m_{\pi}<m_{a_{0}}$. This may explain the lightest quark mass quenched point. In this analysis we used the $a_{0}$ from the local source, or the "1 $\otimes \mathbf{1}$ " operator, which gave the best signal. Within the very large statistical errors, we saw relatively large breaking of flavor symmetry among the different $a_{0}$ channels, with some evidence that this reflects the masses of the different lattice pseudoscalars to which the various $a_{0}$ 's should couple. 


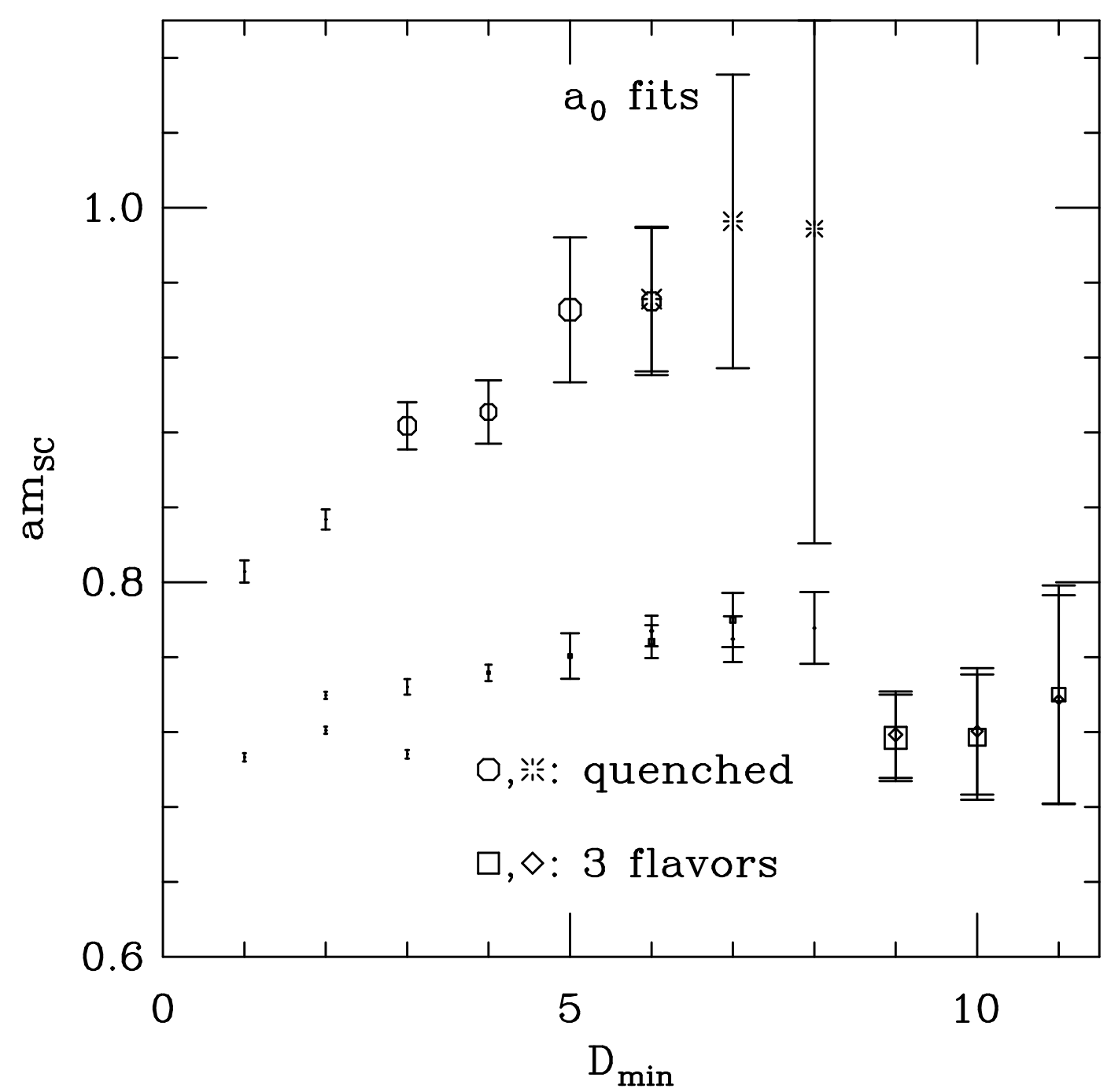

FIG. 14. Fitted masses for the scalar $\left(a_{0}\right)$ meson with quark mass $a m_{u, d}=0.03$ as a function of the minimum distance included in the fit. In this plot the symbol size indicates the confidence level of the fit, with the symbol size used in the legend corresponding to $50 \%$. Here the octagons are quenched results using two $0^{-+}$(pion) states and one $0^{++}\left(a_{0}\right)$ state, while the bursts are quenched results with one state of each parity. The squares and diamonds are $2+1$ flavor results using two and one $0^{-+}$states respectively. 


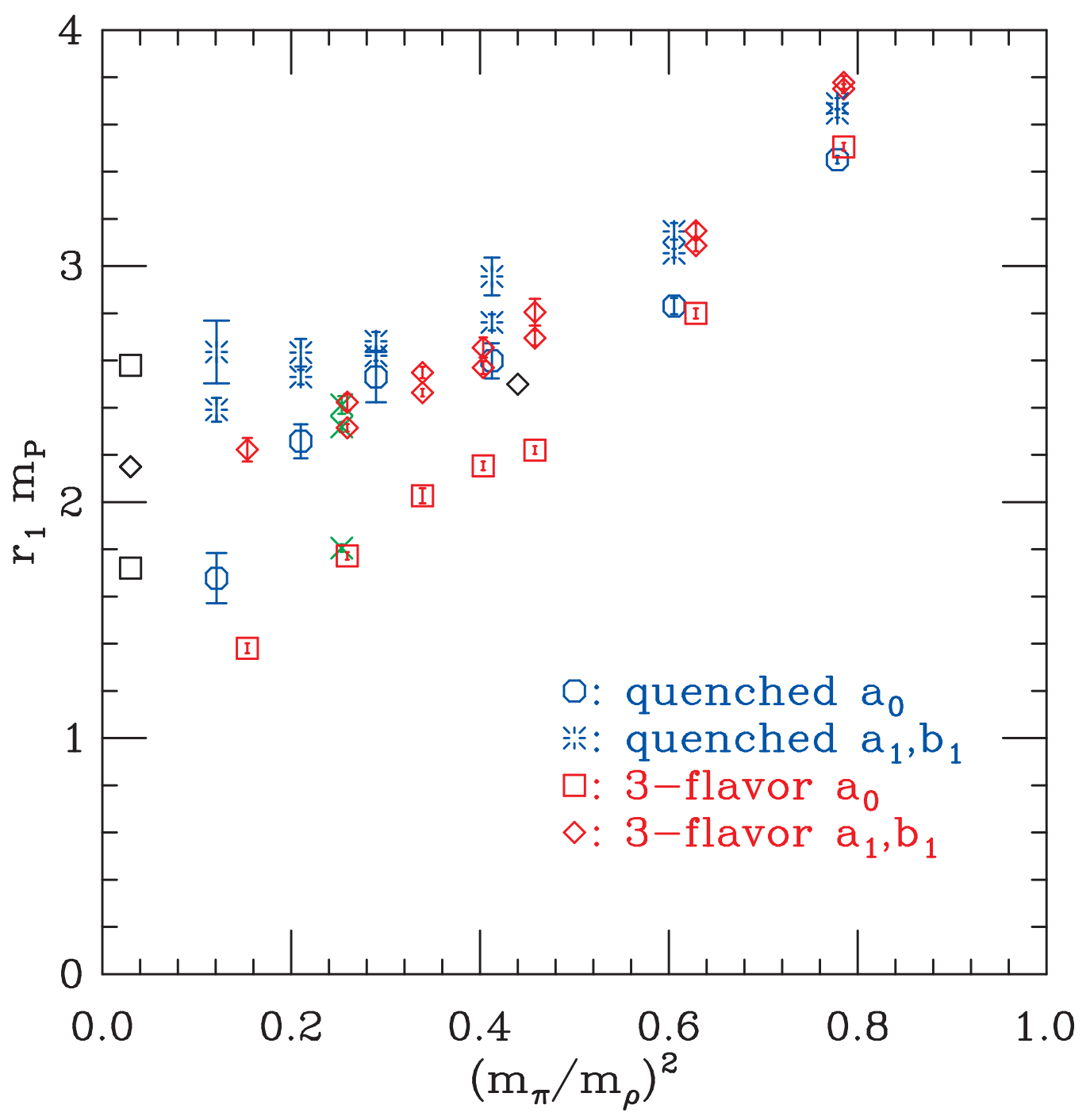

FIG. 15. P-wave meson masses in units of $r_{1}$. The bursts are the quenched pseudovector mesons $\left(a_{1}\right.$ and $\left.b_{1}\right)$, and the diamonds the full QCD pseudovectors. Where the difference is significant the $1^{+-}\left(b_{1}\right)$ is heavier than the $1^{++}$. The octagons are the quenched scalar $\left(a_{0}\right)$, and the squares the full QCD scalar. Crosses are the two flavor results. The diamond at the physical value of $\left(m_{\pi} / m_{\rho}\right)^{2}$ is the experimental $a_{1}$ and $b_{1}$ mass, and the two squares are experimental $0^{++}\left(a_{0}\right)$ masses. 


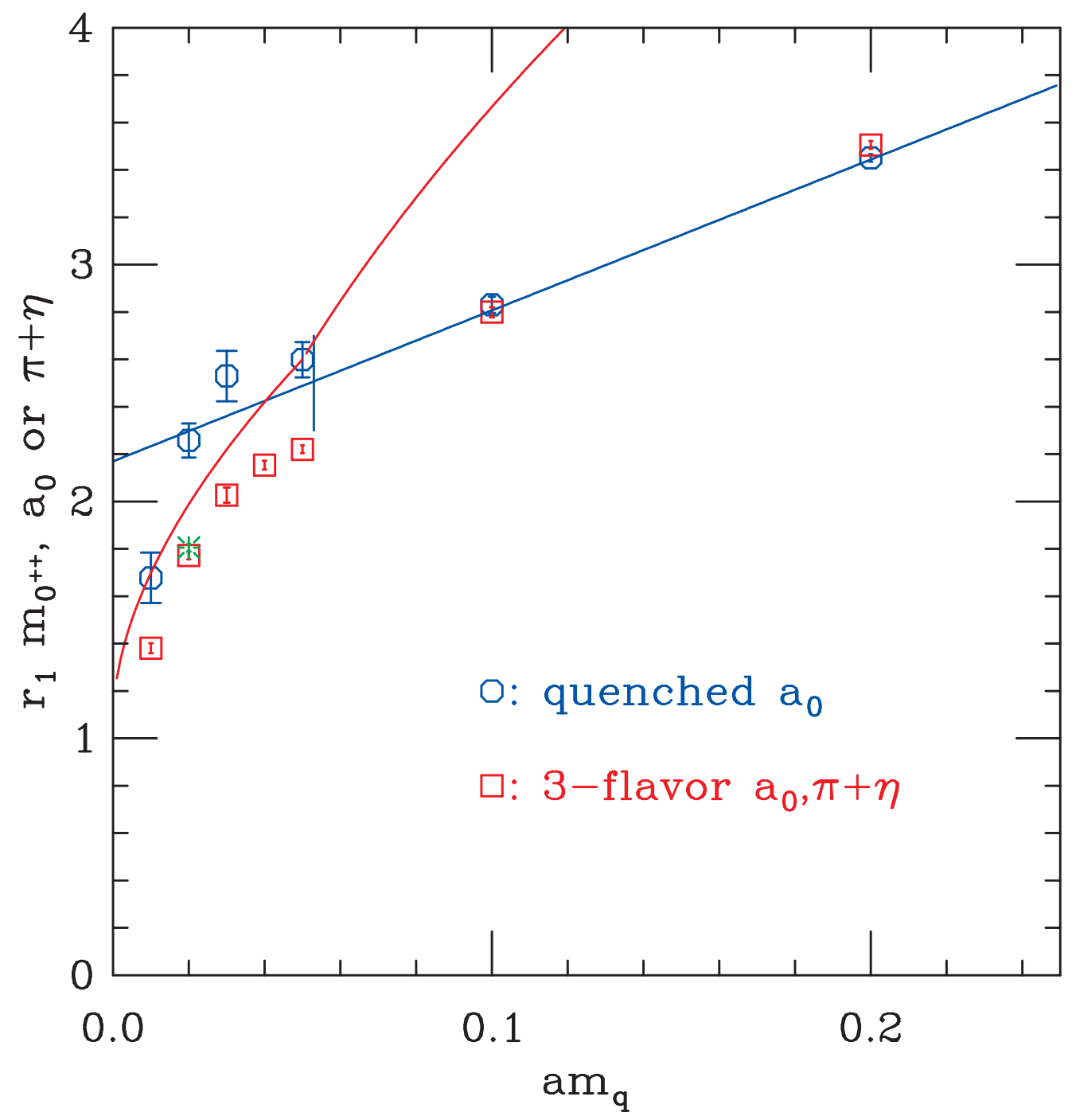

FIG. 16. $0^{++}$masses versus quark mass. The lighest fitted energy in the scalar channel. Octagons are quenched results, squares are three flavor results, and the burst is the two flavor run. The straight line is a crude extrapolation of the heavy quark points. The curved line is the $\pi+\eta$ mass estimate, as discussed in the text. The short vertical line marks the approximate quark mass where the $a_{0}$ mass is twice the quenched pion mass.

\section{Baryons}

We have evaluated propagators for baryons using the "corner wall" source for both degenerate and nondegenerate quarks, using a pointlike sink operator with all three quarks on the same lattice site. With nondegenerate quarks, the lightest states in this channel for zero, one or two strange quarks are the $N$, the $\Lambda$ and the $\Xi$ respectively. However, since we took no special measures to make the operator with one strange quark and two light quarks orthogonal to the $\Sigma$, these propagators undoubtedly contain contamination from a 
nearby $\Sigma$. In order to get the decuplet baryons, we followed Ref. [12], using a wall source on every spatial site, and using the operator in Eq. 6.3 of Ref. [22] for the $\Delta$. This is necessary because the corner wall source does not overlap this $\Delta$ operator. As a byproduct of the calculation of the decuplet mass, we obtain nucleon propagators from even site wall sources and a wall source containing all sites. These propagators are generally noisier than the corner wall source propagators, and the plateau in the effective mass occurs at larger distances. Therefore we generally use the corner source propagators. However, if one uses our fitting procedures on the even-wall or full-wall propagators, one invariably selects a smaller mass than from the corner-wall source propagators. This situation is illustrated by Fig. 17, which show mass fits to these two propagators as a function of the minimum distance included in the fit. In this figure the corner source propagators reach a plateau earlier and with smaller error bars. However, there are perfectly acceptable fits to the full wall source propagators giving masses significantly smaller than the corner source values. Perhaps the only good thing we can say about this situation is that the effect is similar in the quenched and dynamical runs, so as long as we are careful to make the same choices in both cases, we can investigate the effects of sea quarks on the spectrum while taking the statistical errors at face value. The results of the fits that we selected are listed in Tables VIII and IX].

The nucleon to rho mass ratio, or "Edinburgh plot", has long been used as a way of displaying lattice spectrum results. This ratio is known to be sensitive to lattice spacing, lattice volume and quark masses. As mentioned above, we do not address the issues of continuum extrapolation and chiral extrapolation in this paper. However, since our quenched and dynamical lattices are matched in lattice spacing and physical size, we are well positioned to ask if effects of dynamical quarks show up in this ratio. In Fig. 18 we show a variant of the Edinburgh plot, the "APE plot", where $\left(m_{\pi} / m_{\rho}\right)^{2}$ is used as the abscissa, so that for small quark masses the abscissa is proportional to the quark mass. Most of the points on this plot are from the $a \approx 0.13 \mathrm{fm}$ matched lattice runs. It can be seen that there are no significant differences between the quenched and three flavor runs. The single two flavor point lies slightly above the trend, although this is probably not significant. This agreement between $m_{N} / m_{\rho}$ for quenched and full QCD is in apparent conflict with our extrapolations of the conventional action [23]. The discrepancy, which may be due to residual discretization effects in one or both calculations, is under study. We are hopeful that currently running three flavor simulations at finer lattice spacing will shed new light here. Figure 18 also contains a point from a coarser lattice three flavor run and a preliminary point from a finer lattice quenched run $\left(10 / g^{2}=8.4,28^{3} \times 96\right.$ lattice, $\left.a \approx 0.09 \mathrm{fm}\right)$ These two points suggest that when we are in a position to do a continuum extrapolation the continuum results will be lower.

It is interesting to compare these results to the conventional Kogut-Susskind quark action. Figure 19 shows the improved action results together with conventional action results at $6 / g^{2}=5.7,5.85$. 6.15 [24] and 6.5 [25], which correspond to lattice spacings of about $0.16,0.12,0.07$ and $0.043 \mathrm{fm}$ respectively. While a continuum extrapolation will be deferred until the $0.1 \mathrm{fm}$ runs are completed, we can see in this plot that the improved action at 0.13 $\mathrm{fm}$ gives results similar to the conventional action at $a=0.07 \mathrm{fm}$.

Just as for the mesons, one of the biggest problems in comparing baryon masses to the real world is the need for an extrapolation in quark mass. For the mesons the quantity $J$ has the nice feature that it is only minimally sensitive to this extrapolation. It is tempting to 
try to construct similar quantities for the baryons. This suggests looking at the $\Omega^{-}$, the sss decuplet baryon. More generally, we could plot the mass of the decuplet baryon as a function of $\left(m_{P S} / m_{V}\right)^{2}$ to produce a variant of the Edinburgh plot which has the nice feature that there are two experimental points, one for $m_{\Omega^{-}} / m_{\phi}$ and another for $m_{\Delta} / m_{\rho}$. Unfortunately, because the decuplet masses are hard to determine on the lattice, the errors are rather large. The result of this exercise is in Fig. 20. Although the error bars and the scatter among the points are large, the overall trend of this plot is encouraging. It is premature to say whether this plot shows real differences between full and quenched QCD.

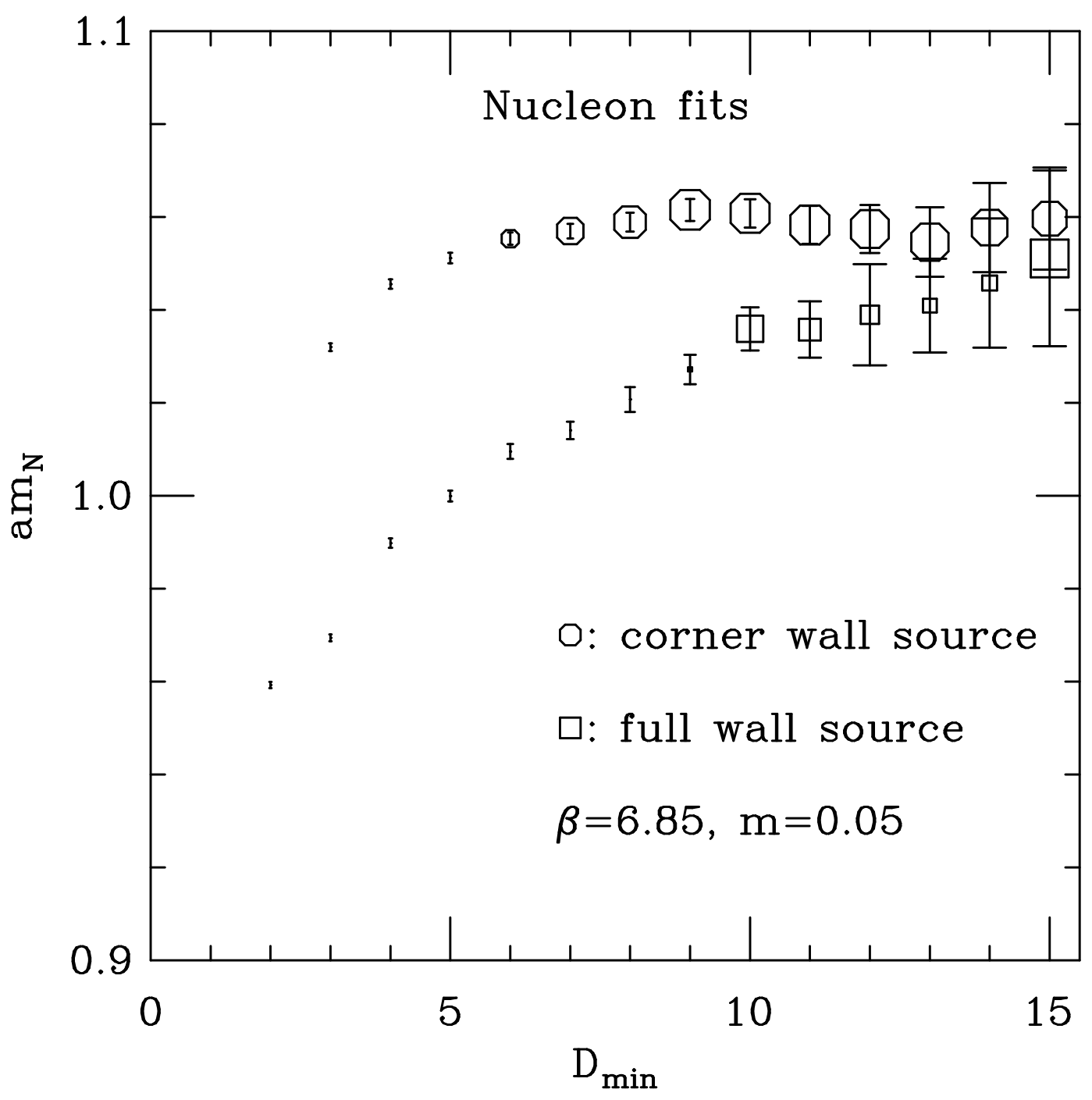


FIG. 17. Mass fits to nucleon propagators as a function of the minimum distance included in the fit. These fits are from the three flavor run with $10 / g^{2}=6.85$ and $a m_{q}=0.05$, and are fairly typical. Here the octagons are from propagators with a "corner wall" source and point sink on all sites, and the squares are from the "full wall" source with a point sink on even sites only. In this plot, the symbol size at each point is proportional to the confidence level of the fit, on a scale where the symbols in the legend correspond to 50\% confidence. For this quark mass we used $D_{\min }=12$ for our quoted mass.

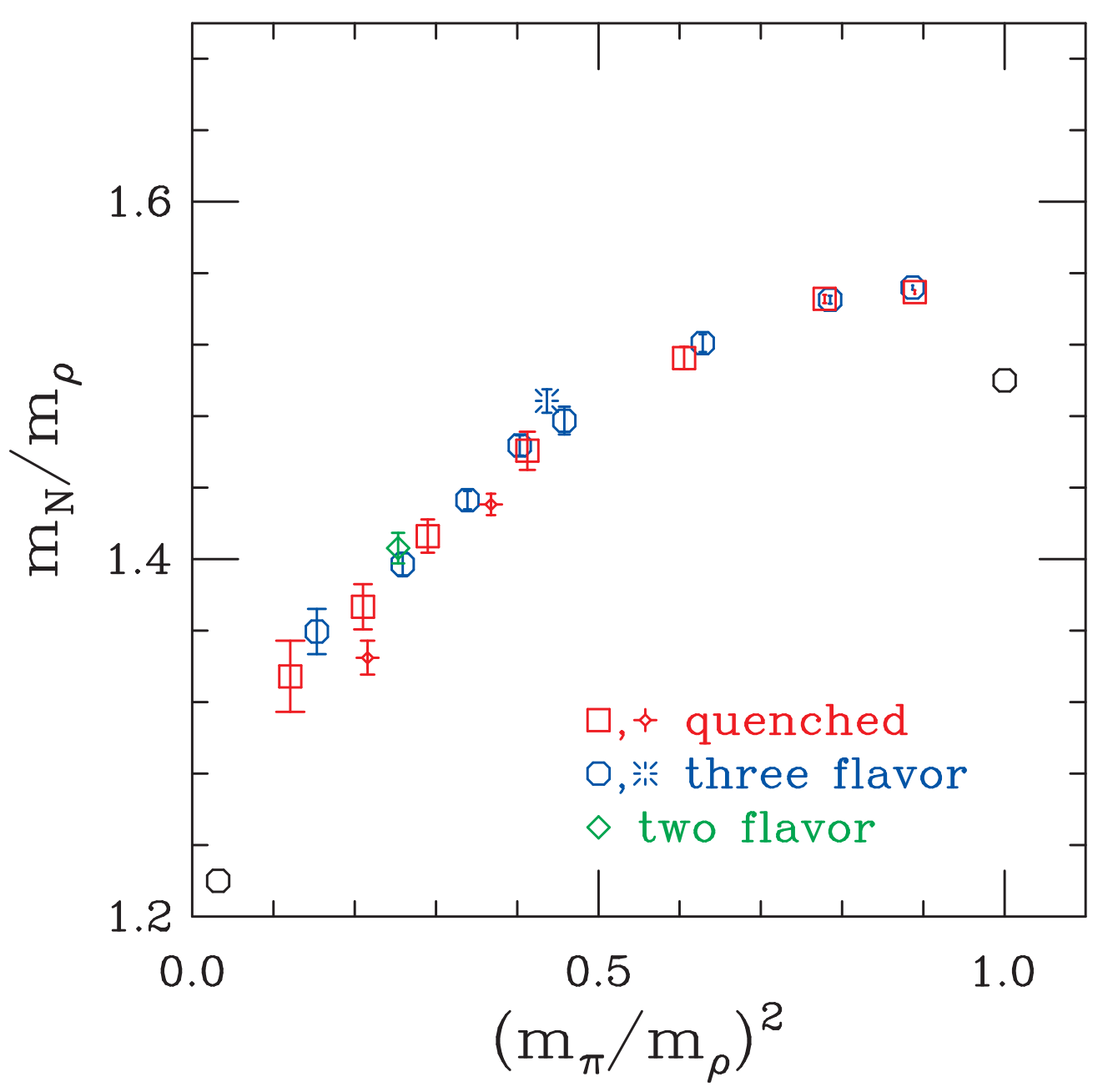

FIG. 18. The nucleon to rho mass ratio in quenched and full QCD. The squares, diamond and octagons are the $a \approx 0.13 \mathrm{fm}$ matched lattice runs with zero, two and three flavors respectively. The fancy diamonds below the other points are preliminary quenched points at $a \approx 0.09 \mathrm{fm}$, and the burst lying above the trend is a coarse lattice three flavor run at $a \approx 0.2 \mathrm{fm}$. The octagon at the left is the physical value, and the octagon at the right is the trivial infinite quark mass value. 


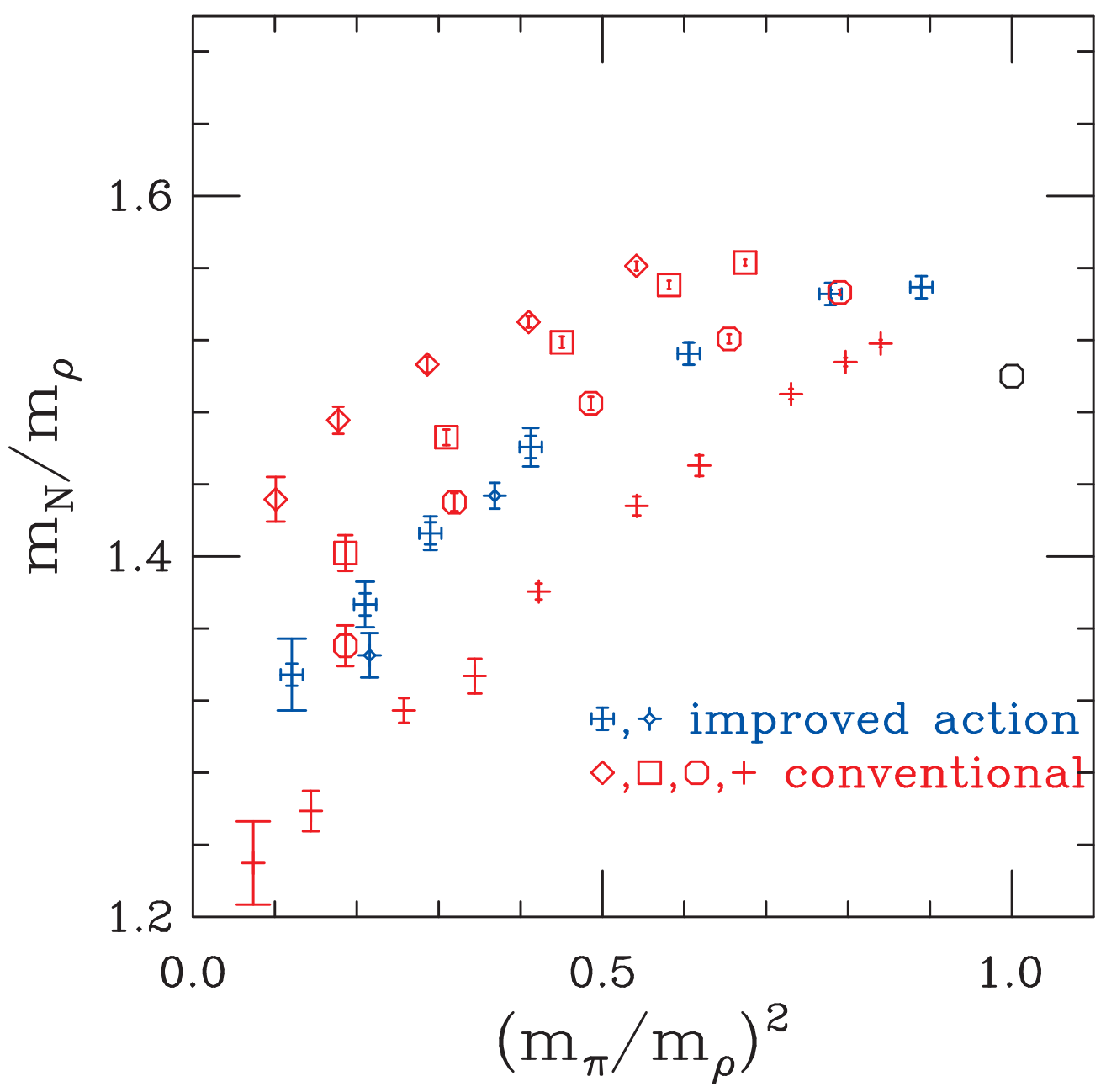

FIG. 19. The nucleon to rho mass ratio in quenched QCD with conventional and improved Kogut-Susskind quark action. The diamonds, squares, octagons and pluses are from the conventional action with lattice spacings of $0.16,0.12,0.07$ and $0.043 \mathrm{fm}$ respectively. The decorated pluses are the improved action results with $a \approx 0.13 \mathrm{fm}$, and the fancy diamonds are preliminary improved action results at $a \approx 0.09 \mathrm{fm}$. 


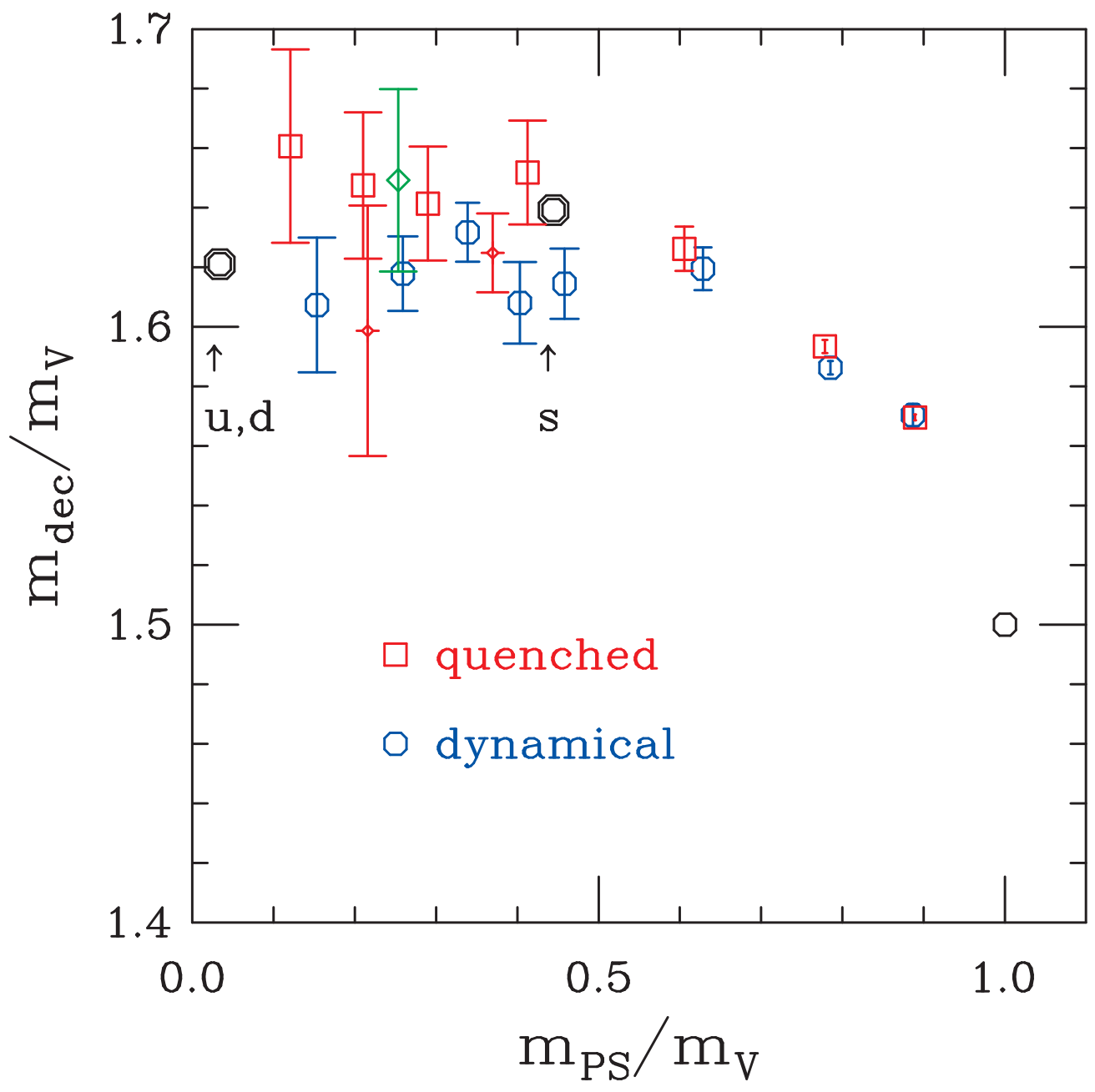

FIG. 20. The decuplet baryon to vector meson mass ratio at $a \approx 0.13 \mathrm{fm}$. Squares are the $a \approx 0.13 \mathrm{fm}$ quenched runs, and octagons the $a \approx 0.13 \mathrm{fm}$ three flavor runs. The single diamond is the $a \approx 0.13 \mathrm{fm}$ two flavor run. The bold octagons without error bars are $m_{\Omega^{-}} / m_{\phi}$ and $m_{\Delta} / m_{\rho}$.

\section{E. Nonzero momentum}

As a check on the quality of the dynamics in our simulations, we calculated the energies of a few nonzero momentum mesons, namely the Goldstone pion with momenta $\frac{2 \pi}{L}(0,0,1)$ and $\frac{2 \pi}{L}(0,1,1)$ and the $\gamma_{z} \otimes \gamma_{z}$ rho with momentum $\frac{2 \pi}{L}(0,0,1)$. We compare these energies

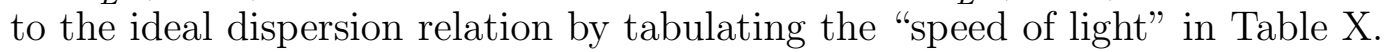

$$
c^{2}=\frac{E(\vec{k})-E(\overrightarrow{0})}{k^{2}}
$$

We see that the dispersion relation is generally very good, but with noticeable deviations from one for the heavier mesons. The results are similar in the quenched and full QCD 
calculations, as can be seen by comparing lines with the same valence quark mass in Table $\mathrm{X}$.

\section{CONCLUSION}

We have used simulations with three flavors of dynamical quarks and a quenched simulation on lattices with matched lattice spacing and physical size to isolate effects of the sea quarks on the hadron spectrum and on the static quark potential. This was done with a Symanzik improved gauge action and an improved Kogut-Susskind quark action to make the effects of the nonzero lattice spacing as small as practical. Effects of the sea quarks are clearly visible in the static potential. The mesonic mass ratio $J$ is much closer to the experimental value when dynamical quarks are included. The $a_{0}$ meson couples strongly to two meson states, as expected when sea quarks are included.

Several aspects need further study. While the quenched pion mass has the expected form, we have not understood the dependence of the three flavor pion mass on the quark masses. It would be very nice to be able to extract excited state masses, especially in the avoided level crossing of the $a_{0}$, and to extract decay rates to be compared to experiment. Although we have minimized the lattice artifacts by using an improved action, an empirical investigation of these effects is necessary. We are beginning a series of simulations with $a \approx 0.09 \mathrm{fm}$ to investigate these effects.

\section{ACKNOWLEDGEMENTS}

Computations were done on the Origin 2000 clusters at LANL, on the T3E and SP3 at NERSC, on the T3E, SP2 and SP3 at SDSC, on the NT and Linux clusters and Origin 2000 at NCSA, on the Origin 2000 at BU, on the Roadrunner and Los Lobos clusters at UNM and on the PC clusters at NERSC, Indiana University and the University of Utah. This work was supported by the U.S. Department of Energy under contracts DOE - DEFG02-91ER-40628, DOE - DE-FG03-95ER-40894, DOE - DE-FG02-91ER-40661, DOE DE-FG02-97ER-41022 and DOE - DE-FG03-95ER-40906 and National Science Foundation grants NSF - PHY99-70701 and NSF - PHY97-22022. 


\section{REFERENCES}

[1] The MILC Collaboration: C. Bernard et al., Phys. Rev. D 58 (1998) 014503; The MILC Collaboration: T. Blum et al., Phys. Rev. D 55, 1133 (1997); G.P. Lepage, Nucl. Phys. (Proc. Suppl.) 60A, 267 (1998); K. Orginos and D. Toussaint, Phys. Rev. D 59 (1999) 014501; Nucl. Phys. B (Proc. Suppl.) 73, 909 (1999); J.F. Lagäe and D.K. Sinclair, Nucl. Phys. (Proc. Suppl.) 63, 892 (1998); Phys. Rev. D 59 (1999) 014511; K. Orginos, D. Toussaint and R.L. Sugar, Phys. Rev. D 60 (1999) 054503; Nucl. Phys. B (Proc. Suppl.) 83-84, 878 (2000); G.P. Lepage, Phys. Rev. D 59 (1999) 074501.

[2] K. Symanzik, in "Recent Developments in Gauge Theories", eds. G. 't Hooft et al., 313 (Plenum, New York, 1980); Nucl. Phys. B226 187 (1983); M. Lüscher and P. Weisz, Comm. Math. Phys. 9719 (1985); Phys. Lett. 158B 250 (1985); G. P. Lepage and P. B. Mackenzie, Phys. Rev. D48 2250 (1993); M. Alford, W. Dimm, G.P. Lepage, G. Hockney and P.B. Mackenzie, Phys. Lett. 361B 87 (1995).

[3] The MILC collaboration: C. Bernard et al., Phys. Rev. D 61, 111502 (2000).

[4] P. Lacock and C. Michael, Phys. Rev. D 525213 (1995).

[5] K. Kanaya, hep-lat/0009263, Talk presented at the XXXth International Conference on High Energy Physics (ICHEP 2000), July 27-August 2, 2000, Osaka, Japan; CP-PACS collaboration: A. Ali Khan et al., hep-lat/0010078, to appear in Nucl. Phys. B (Proc. Suppl.).

[6] JLQCD collaboration: A. Aoki et al., hep-lat/0010086, to appear in Nucl. Phys. B (Proc. Suppl.).

[7] S. Gottlieb et al., Phys. Rev. D 35, 2531 (1987).

[8] S. Aoki, Nucl. Phys. B (Proc. Suppl.) 94, 3 (2001).

[9] The MILC Collaboration: C. Bernard et al., Phys. Rev. D 62, 034503 (2000).

[10] R. Sommer, Nucl. Phys. B411, 839 (1994).

[11] UKQCD Collaboration, S.P. Booth et al., Phys. Lett. B294, 385 (1992).

[12] R. Gupta, G. Guralnik, C. Kilcup and S. Sharpe, Phys. Rev. D 43, 2003 (1991).

[13] M.F.L. Golterman and J. Smit, Nucl. Phys. B255 328 (1985); M.F.L. Golterman, Nucl. Phys. B273, 663 (1986).

[14] W. Lee and S. Sharpe, Phys. Rev. D 60 (1999) 114503.

[15] CP-PACS collaboration: T. Manke et al., Phys. Rev. D 62 (2000) 114508.

[16] C. Bernard and M. Golterman, Phys. Rev. D bf 46, 853 (1992).

[17] S.R. Sharpe, Phys. Rev. D bf 46, 3146 (1992).

[18] S.Aoki et al., Phys.Rev.Lett. 84, 238 (2000).

[19] W. Bardeen et al., Phys.Rev. D 62, 114505 (200).

[20] J. Gasser and H. Leutwyler, Nucl. Phys. B250, 465 (1985).

[21] C. Bernard and M. Golterman, Phys. Rev. D 53, 476 (1996).

[22] M.F.L. Golterman and J. Smit, Nucl. Phys. B255, 328 (1985).

[23] The MILC Collaboration: C. Bernard et al., Nucl. Phys. B (Proc. Suppl.) 73, 198 (1999); Phys. Rev. Lett. 81, 3087 (1998).

[24] The MILC Collaboration: C. Bernard et al., Nucl. Phys. B (Proc. Suppl.) 60A, 3 (1998)

[25] S. Kim and S. Ohta, Phys. Rev. D 61 (2000) 074506. 


\section{TABLES}

\begin{tabular}{|l|c|l|l|l|l|l|}
\hline \hline$a m_{u, d} / a m_{s}$ & $10 / g^{2}$ & $u_{0}$ & res. & $\epsilon$ & lats. & $a / r_{1}$ \\
\hline quenched & 8.00 & 0.8879 & na & na & 408 & $0.3762(8)$ \\
\hline $0.02 /$ na & 7.20 & 0.8755 & $1 \times 10^{-4}$ & 0.013 & 370 & $0.3745(14)$ \\
\hline $0.40 / 0.40$ & 7.35 & 0.8822 & $2 \times 10^{-5}$ & 0.03 & 332 & $0.3766(10)$ \\
$0.20 / 0.20$ & 7.15 & 0.8787 & $5 \times 10^{-5}$ & 0.03 & 341 & $0.3707(10)$ \\
$0.10 / 0.10$ & 6.96 & 0.8739 & $5 \times 10^{-5}$ & 0.03 & 339 & $0.3730(14)$ \\
$0.05 / 0.05$ & 6.85 & 0.8707 & $1 \times 10^{-4}$ & 0.02 & 425 & $0.3742(15)$ \\
$0.04 / 0.05$ & 6.83 & 0.8702 & $5 \times 10^{-5}$ & 0.02 & 351 & $0.3765(14)$ \\
$0.03 / 0.05$ & 6.81 & 0.8696 & $5 \times 10^{-5}$ & 0.02 & 564 & $0.3775(12)$ \\
$0.02 / 0.05$ & 6.79 & 0.8688 & $1 \times 10^{-4}$ & 0.0133 & 484 & $0.3775(12)$ \\
$0.01 / 0.05$ & 6.76 & 0.8677 & $1 \times 10^{-4}$ & 0.00667 & 407 & $0.3852(14)$ \\
\hline \hline
\end{tabular}

TABLE I. Parameters of the $a=0.13$ fm simulations

\begin{tabular}{|c|c|cccccccc|}
\hline \hline & & 0.01 & 0.02 & 0.03 & 0.04 & 0.05 & 0.1 & 0.2 & 0.4 \\
$\mathrm{H}$ & fit & & & & & & & & \\
$\pi$ & 1,0 & 18 & 18 & 18 & 18 & 18 & 18 & 18 & 18 \\
$K$ & 1,0 & 14 & 14 & 14 & 14 & & & & \\
$s \bar{s}$ & 1,0 & 14 & 14 & 14 & 14 & & & & \\
$a_{0}$ & 2,1 & 4 & 4 & 5 & 5 & 6 & 6 & 6 & 6 \\
$\rho$ & 1,1 & 6 & 7 & 8 & 9 & 9 & 10 & 12 & 14 \\
$K^{*}$ & 1,1 & 8 & 8 & 9 & 9 & & & & \\
$\phi$ & 1,1 & 8 & 8 & 9 & 9 & & & & \\
$a_{1}$ & 2,1 & 4 & 4 & 4 & 5 & 5 & 5 & 6 & 7 \\
$b_{1}$ & 2,1 & 4 & 4 & 4 & 5 & 5 & 5 & 6 & 18 \\
$N$ & 1,1 & 7 & 8 & 9 & 10 & 12 & 14 & 16 & 18 \\
$\Lambda$ & 1,1 & 7 & 7 & 8 & 9 & & & & \\
$\Xi$ & 1,1 & 7 & 7 & 8 & 9 & & & & \\
$\Delta$ & 1,1 & 3 & 4 & 5 & 6 & 7 & 8 & 9 & 10 \\
\hline \hline
\end{tabular}

TABLE II. Minimum distances used in propagator fits. With the exception of the $\Delta$, these hadrons are obtained from the "corner" source. The top row is the light quark mass. The second column shows the type of fit used, where the two numbers are the number of simple exponentials included and the number of oscillating contributions included. For example, a fit of type "2,1" would include two particles with one parity and one particle with the opposite parity. Hadrons with nondegenerate valence quarks, such as the $K$, were computed only for $m_{u, d}<m_{s}$. 


\begin{tabular}{|llllll}
\hline \hline am valence & $a m_{\text {sea }}$ & $a m_{P S}$ & range & $\chi^{2} / D$ & conf. \\
\hline $0.4(\pi)$ & $\infty$ & $1.45664(12)$ & $18-31$ & $13 / 12$ & 0.39 \\
$0.2(\pi)$ & $\infty$ & $0.96166(16)$ & $18-31$ & $14 / 12$ & 0.32 \\
$0.1(\pi)$ & $\infty$ & $0.65693(19)$ & $18-31$ & $14 / 12$ & 0.28 \\
$0.05(\pi)$ & $\infty$ & $0.46043(20)$ & $18-31$ & $15 / 12$ & 0.25 \\
$0.03(\pi)$ & $\infty$ & $0.35825(16)$ & $18-31$ & $21 / 12$ & 0.05 \\
$0.02(\pi)$ & $\infty$ & $0.29440(17)$ & $18-31$ & $23 / 12$ & 0.026 \\
$0.01(\pi)$ & $\infty$ & $0.21104(16)$ & $18-31$ & $26 / 12$ & 0.01 \\
$0.03 / 0.05(K)$ & $\infty$ & $0.41261(19)$ & $14-31$ & $26 / 16$ & 0.06 \\
$0.02 / 0.05(K)$ & $\infty$ & $0.38650(20)$ & $14-31$ & $25 / 16$ & 0.07 \\
$0.01 / 0.05(K)$ & $\infty$ & $0.35852(23)$ & $14-31$ & $23 / 16$ & 0.13 \\
\hline $0.02(\pi)$ & 0.02 & $0.30258(22)$ & $18-31$ & $9.4 / 12$ & 0.67 \\
$0.02 / 0.05(K)$ & 0.02 & $0.39823(25)$ & $14-31$ & $33 / 16$ & 0.0076 \\
$0.05(s \bar{s})$ & 0.02 & $0.47623(25)$ & $14-31$ & $27 / 16$ & 0.047 \\
\hline $0.4(\pi)$ & 0.4 & $1.46932(17)$ & $18-31$ & $5.3 / 12$ & 0.95 \\
$0.2(\pi)$ & 0.2 & $0.97930(25)$ & $18-31$ & $8.3 / 12$ & 0.76 \\
$0.1(\pi)$ & 0.1 & $18-31$ & $16 / 12$ & 0.17 \\
$0.05(\pi)$ & $0.08332(24)$ & $18-31$ & $26 / 12$ & 0.011 \\
$0.04(\pi)$ & $0.48422(21)$ & $18-31$ & $14 / 12$ & 0.31 \\
$0.03(\pi)$ & $0.04 / 0.05$ & $0.43507(27)$ & $18-31$ & $15 / 12$ & 0.25 \\
$0.02(\pi)$ & $0.03 / 0.05$ & $0.37787(18)$ & $18-31$ & $16 / 12$ & 0.21 \\
$0.01(\pi)$ & $0.02 / 0.05$ & $0.31125(16)$ & $18-31$ & $14 / 12$ & 0.27 \\
$0.04 / 0.05(K)$ & $0.01 / 0.05$ & $0.22446(22)$ & $14-31$ & $18 / 16$ & 0.31 \\
$0.03 / 0.05(K)$ & $0.04 / 0.05$ & $0.46141(27)$ & $14-31$ & $26 / 16$ & 0.052 \\
$0.02 / 0.05(K)$ & $0.03 / 0.05$ & $0.43613(19)$ & $14-31$ & $19 / 16$ & 0.28 \\
$0.01 / 0.05(K)$ & $0.02 / 0.05$ & $0.40984(21)$ & $14-31$ & $25 / 16$ & 0.072 \\
$0.05(s \bar{s})$ & $0.01 / 0.05$ & $0.38334(29)$ & $14-31$ & $18 / 16$ & 0.35 \\
$0.05(s \bar{s})$ & $0.04 / 0.05$ & $0.48659(27)$ & $14-31$ & $28 / 16$ & 0.035 \\
$0.05(s \bar{s})$ & $0.03 / 0.05$ & $0.48796(18)$ & $14-31$ & $23 / 16$ & 0.12 \\
$0.05(s \bar{s})$ & $0.02 / 0.05$ & $0.49009(20)$ & $14-31$ & $19 / 16$ & 0.26 \\
\hline \hline
\end{tabular}

TABLE III. Pseudoscalar meson masses. Quenched results are first, followed by the single two-flavor run, followed by the three flavor runs. The first column is the valence quark mass(es), and the second column the sea quark mass or masses. The particle name in the first column is intended as a mnemonic. Here " $\pi$ " indicates valence quark mass equal to the lighter dynamical quarks, or degenerate in the quenched case. " $K$ " indicates one valence quark equal to the light dynamical quarks and one at about $m_{s}$, while " $s \vec{s}$ " indicates two valence quarks with mass about $m_{s}$, in a flavor nonsinglet state. The remaining columns are the hadron mass, the time range for the chosen fit, $\chi^{2}$ and number of degrees of freedom for the fit, and the confidence level of the fit. 


\begin{tabular}{|c|c|c|c|c|c|}
\hline$a_{\text {valence }}$ & $a m_{\text {sea }}$ & $a m_{V}$ & range & $\chi^{2} / D$ & conf. \\
\hline $0.4(\rho)$ & $\infty$ & $1.5446(2)$ & $14-31$ & $18 / 14$ & 0.19 \\
\hline $0.2(\rho)$ & $\infty$ & $1.0900(5)$ & $12-31$ & $19 / 16$ & 0.27 \\
\hline $0.1(\rho)$ & $\infty$ & $0.8443(11)$ & $10-28$ & $21 / 15$ & 0.15 \\
\hline $0.05(\rho)$ & $\infty$ & $0.7168(21)$ & $9-27$ & $10 / 15$ & 0.8 \\
\hline $0.03(\rho)$ & $\infty$ & $0.6653(26)$ & $8-23$ & $6.9 / 12$ & 0.86 \\
\hline $0.02(\rho)$ & $\infty$ & $0.6422(30)$ & $7-21$ & $7 / 11$ & 0.8 \\
\hline $0.01(\rho)$ & $\infty$ & $0.6070(60)$ & $6-16$ & $10 / 7$ & 0.19 \\
\hline $0.03 / 0.05\left(K^{*}\right)$ & $\infty$ & $0.6910(30)$ & $9-24$ & $13 / 12$ & 0.38 \\
\hline $0.02 / 0.05\left(K^{*}\right)$ & $\infty$ & $0.6820(30)$ & $8-21$ & $10 / 10$ & 0.42 \\
\hline $0.01 / 0.05\left(K^{*}\right)$ & $\infty$ & $0.6680(40)$ & $8-21$ & $11 / 10$ & 0.34 \\
\hline $0.02(\rho)$ & 0.02 & $0.6009(23)$ & $7-23$ & $17 / 13$ & 0.2 \\
\hline $0.02 / 0.05\left(K^{*}\right)$ & 0.02 & $0.6532(23)$ & $8-27$ & $22 / 16$ & 0.14 \\
\hline $0.05(\phi)$ & 0.02 & $0.7003(14)$ & $8-29$ & $23 / 18$ & 0.21 \\
\hline $0.4(\rho)$ & 0.4 & $1.5602(2)$ & $14-31$ & $12 / 14$ & 0.64 \\
\hline $0.2(\rho)$ & 0.2 & $1.1051(7)$ & $12-31$ & $14 / 16$ & 0.56 \\
\hline $0.1(\rho)$ & 0.1 & $0.8620(9)$ & $10-31$ & $16 / 18$ & 0.57 \\
\hline $0.05(\rho)$ & 0.05 & $0.7154(17)$ & $9-31$ & $11 / 19$ & 0.91 \\
\hline $0.04(\rho)$ & $0.04 / 0.05$ & $0.6853(17)$ & $9-28$ & $11 / 16$ & 0.83 \\
\hline $0.03(\rho)$ & $0.03 / 0.05$ & $0.6490(14)$ & $8-27$ & $19 / 16$ & 0.26 \\
\hline $0.02(\rho)$ & $0.02 / 0.05$ & $0.6113(19)$ & $7-22$ & $13 / 12$ & 0.38 \\
\hline $0.01(\rho)$ & $0.01 / 0.05$ & $0.5730(30)$ & $6-18$ & $7.4 / 9$ & 0.59 \\
\hline $0.04 / 0.05\left(K^{*}\right)$ & $0.04 / 0.05$ & $0.7040(22)$ & $9-29$ & $15 / 17$ & 0.61 \\
\hline $0.03 / 0.05\left(K^{*}\right)$ & $0.03 / 0.05$ & $0.6845(18)$ & $9-29$ & $21 / 17$ & 0.22 \\
\hline $0.02 / 0.05\left(K^{*}\right)$ & $0.02 / 0.05$ & $0.6631(19)$ & $8-25$ & $15 / 14$ & 0.38 \\
\hline $0.01 / 0.05\left(K^{*}\right)$ & $0.01 / 0.05$ & $0.6485(28)$ & $8-23$ & $4.7 / 12$ & 0.97 \\
\hline $0.05(\phi)$ & $0.04 / 0.05$ & $0.7198(19)$ & $9-29$ & $13 / 17$ & 0.77 \\
\hline $0.05(\phi)$ & $0.03 / 0.05$ & $0.7174(13)$ & $9-29$ & $19 / 17$ & 0.35 \\
\hline $0.05(\phi)$ & $0.02 / 0.05$ & $0.7152(11)$ & $8-29$ & $21 / 18$ & 0.28 \\
\hline $0.05(\phi)$ & $0.01 / 0.05$ & $0.7194(11)$ & $8-28$ & $11 / 17$ & 0.84 \\
\hline
\end{tabular}

TABLE IV. Vector meson masses. The format is the same as Table III. 


\begin{tabular}{|llllll|}
\hline \hline$a m_{\text {valence }}$ & $a m_{\text {sea }}$ & $a m_{S C}$ & range & $\chi^{2} / D$ & conf. \\
\hline $0.4\left(a_{0}\right)$ & $\infty$ & $1.739(4)$ & $6-20$ & $11 / 9$ & 0.29 \\
$0.2\left(a_{0}\right)$ & $\infty$ & $1.296(6)$ & $6-20$ & $5 / 9$ & 0.83 \\
$0.1\left(a_{0}\right)$ & $\infty$ & $1.063(13)$ & $6-20$ & $12 / 9$ & 0.24 \\
$0.05\left(a_{0}\right)$ & $\infty$ & $0.976(28)$ & $6-31$ & $22 / 22$ & 0.48 \\
$0.03\left(a_{0}\right)$ & $\infty$ & $0.950(40)$ & $5-20$ & $9 / 10$ & 0.52 \\
$0.02\left(a_{0}\right)$ & $\infty$ & $0.848(27)$ & $4-20$ & $10 / 11$ & 0.51 \\
$0.01\left(a_{0}\right)$ & $\infty$ & $0.630(40)$ & $4-20$ & $17 / 12$ & 0.12 \\
\hline $0.02\left(a_{0}\right)$ & 0.02 & $0.676(6)$ & $4-31$ & $25 / 24$ & 0.41 \\
\hline $0.4\left(a_{0}\right)$ & 0.4 & $1.750(4)$ & $6-20$ & $21 / 9$ & 0.013 \\
$0.2\left(a_{0}\right)$ & 0.2 & $1.297(6)$ & $6-20$ & $4.8 / 9$ & 0.85 \\
$0.1\left(a_{0}\right)$ & 0.1 & $1.042(8)$ & $6-20$ & $18 / 9$ & 0.031 \\
$0.05\left(a_{0}\right)$ & 0.05 & $0.829(6)$ & $5-31$ & $63 / 23$ & $1.3 \mathrm{e}-05$ \\
$0.04\left(a_{0}\right)$ & $0.04 / 0.05$ & $0.808(7)$ & $5-20$ & $10 / 10$ & 0.41 \\
$0.03\left(a_{0}\right)$ & $0.03 / 0.05$ & $0.761(12)$ & $5-20$ & $16 / 10$ & 0.094 \\
$0.02\left(a_{0}\right)$ & $0.02 / 0.05$ & $0.669(6)$ & $4-20$ & $31 / 11$ & 0.0011 \\
$0.01\left(a_{0}\right)$ & $0.01 / 0.05$ & $0.532(8)$ & $4-20$ & $15 / 11$ & 0.2 \\
\hline \hline
\end{tabular}

TABLE V. $0^{++}\left(a_{0}\right)$ meson masses. The format is the same as Table [II].

\begin{tabular}{|llllll|}
\hline \hline$a m_{\text {valence }}$ & $a m_{\text {sea }}$ & $a m_{P V}$ & range & $\chi^{2} / D$ & conf. \\
\hline $0.4\left(a_{1}\right)$ & $\infty$ & $1.816(7)$ & $7-20$ & $12 / 8$ & 0.17 \\
$0.2\left(a_{1}\right)$ & $\infty$ & $1.370(7)$ & $6-20$ & $4.7 / 9$ & 0.86 \\
$0.1\left(a_{1}\right)$ & $\infty$ & $1.147(7)$ & $5-20$ & $3.6 / 10$ & 0.96 \\
$0.05\left(a_{1}\right)$ & $\infty$ & $1.037(13)$ & $5-20$ & $7.7 / 10$ & 0.65 \\
$0.03\left(a_{1}\right)$ & $\infty$ & $0.984(8)$ & $4-20$ & $15 / 11$ & 0.17 \\
$0.02\left(a_{1}\right)$ & $\infty$ & $0.950(12)$ & $4-18$ & $21 / 9$ & 0.013 \\
$0.01\left(a_{1}\right)$ & $\infty$ & $0.898(19)$ & $4-16$ & $21 / 7$ & 0.39 \\
\hline $0.02\left(a_{1}\right)$ & 0.02 & $0.868(8)$ & $4-20$ & $11 / 11$ & 0.43 \\
\hline $0.4\left(a_{1}\right)$ & 0.4 & $1.826(8)$ & $7-20$ & $12 / 8$ & 0.17 \\
$0.2\left(a_{1}\right)$ & 0.2 & $1.388(7)$ & $6-20$ & $12 / 9$ & 0.2 \\
$0.1\left(a_{1}\right)$ & 0.1 & $1.149(9)$ & $5-20$ & $7.3 / 10$ & 0.7 \\
$0.05\left(a_{1}\right)$ & 0.05 & $1.006(12)$ & $5-20$ & $28 / 10$ & 0.0018 \\
$0.04\left(a_{1}\right)$ & $0.04 / 0.05$ & $0.964(10)$ & $5-20$ & $9.6 / 10$ & 0.47 \\
$0.03\left(a_{1}\right)$ & $0.03 / 0.05$ & $0.925(6)$ & $4-20$ & $7.2 / 11$ & 0.78 \\
$0.02\left(a_{1}\right)$ & $0.02 / 0.05$ & $0.874(6)$ & $4-20$ & $10 / 11$ & 0.53 \\
$0.01\left(a_{1}\right)$ & $0.01 / 0.05$ & $8.131(11)$ & $4-15$ & $2.6 / 6$ & 0.86 \\
\hline \hline
\end{tabular}

TABLE VI. $1^{++}\left(a_{1}\right)$ meson masses. The format is the same as Table [II. 


\begin{tabular}{|llllll|}
\hline \hline$a m_{\text {valence }}$ & $a m_{\text {sea }}$ & $a m_{P V}$ & range & $\chi^{2} / D$ & conf. \\
\hline $0.4\left(b_{1}\right)$ & $\infty$ & $1.833(9)$ & $7-20$ & $13 / 8$ & 0.11 \\
$0.2\left(b_{1}\right)$ & $\infty$ & $1.385(9)$ & $6-20$ & $8.1 / 9$ & 0.53 \\
$0.1\left(b_{1}\right)$ & $\infty$ & $1.182(13)$ & $5-20$ & $12 / 10$ & 0.29 \\
$0.05\left(b_{1}\right)$ & $\infty$ & $1.110(30)$ & $5-20$ & $12 / 10$ & 0.3 \\
$0.03\left(b_{1}\right)$ & $\infty$ & $1.007(15)$ & $4-20$ & $16 / 11$ & 0.16 \\
$0.02\left(b_{1}\right)$ & $\infty$ & $0.989(22)$ & $4-20$ & $13 / 11$ & 0.28 \\
$0.01\left(b_{1}\right)$ & $\infty$ & $0.990(50)$ & $4-16$ & $8.9 / 7$ & 0.26 \\
\hline $0.02\left(b_{1}\right)$ & 0.02 & $0.903(14)$ & $4-20$ & $11 / 11$ & 0.43 \\
\hline $0.4\left(b_{1}\right)$ & 0.4 & $1.834(12)$ & $7-20$ & $10 / 8$ & 0.26 \\
$0.2\left(b_{1}\right)$ & 0.2 & $1.398(10)$ & $6-20$ & $7 / 9$ & 0.64 \\
$0.1\left(b_{1}\right)$ & 0.1 & $1.172(12)$ & $5-20$ & $7.9 / 10$ & 0.64 \\
$0.05\left(b_{1}\right)$ & 0.05 & $1.047(21)$ & $5-20$ & $2.5 / 10$ & 0.99 \\
$0.04\left(b_{1}\right)$ & $0.04 / 0.05$ & $0.996(16)$ & $5-20$ & $11 / 10$ & 0.39 \\
$0.03\left(b_{1}\right)$ & $0.03 / 0.05$ & $0.957(9)$ & $4-20$ & $9.5 / 11$ & 0.58 \\
$0.02\left(b_{1}\right)$ & $0.02 / 0.05$ & $0.915(13)$ & $4-20$ & $13 / 11$ & 0.3 \\
$0.01\left(b_{1}\right)$ & $0.01 / 0.05$ & $0.856(19)$ & $4-18$ & $8.7 / 9$ & 0.47 \\
\hline \hline
\end{tabular}

TABLE VII. $1^{+-}\left(b_{1}\right)$ meson masses. The format is the same as Table [II]. 


\begin{tabular}{|c|c|c|c|c|c|}
\hline$a_{\text {valence }}$ & $a m_{\text {sea }}$ & $a m_{B}$ & range & $\chi^{2} / D$ & conf. \\
\hline $0.4(\mathrm{~N})$ & $\infty$ & $2.3933(15)$ & $18-31$ & $21 / 10$ & 0.021 \\
\hline $0.2(\mathrm{~N})$ & $\infty$ & $1.6847(23)$ & $16-31$ & $19 / 12$ & 0.084 \\
\hline $0.1(\mathrm{~N})$ & $\infty$ & $1.2770(50)$ & $14-30$ & $15 / 13$ & 0.33 \\
\hline $0.05(\mathrm{~N})$ & $\infty$ & $1.0470(70)$ & $12-21$ & $2.9 / 6$ & 0.82 \\
\hline $0.03(\mathrm{~N})$ & $\infty$ & $0.9400(50)$ & $9-19$ & $8.1 / 7$ & 0.32 \\
\hline $0.02(\mathrm{~N})$ & $\infty$ & $0.8820(70)$ & $8-17$ & $5.3 / 6$ & 0.5 \\
\hline $0.01(\mathrm{~N})$ & $\infty$ & $0.8100(90)$ & $7-14$ & $6.8 / 4$ & 0.15 \\
\hline $0.03 / 0.05(\Lambda)$ & $\infty$ & $0.9730(40)$ & 8-19 & $5.7 / 8$ & 0.68 \\
\hline $0.02 / 0.05(\Lambda)$ & $\infty$ & $0.9310(40)$ & $7-19$ & $7.1 / 9$ & 0.63 \\
\hline $0.01 / 0.05(\Lambda)$ & $\infty$ & $0.8900(60)$ & $7-15$ & $11 / 5$ & 0.05 \\
\hline $0.03 / 0.05(\Xi)$ & $\infty$ & $1.0090(30)$ & $8-20$ & $6.4 / 9$ & 0.7 \\
\hline $0.02 / 0.05(\Xi)$ & $\infty$ & $0.9867(28)$ & $7-20$ & $6.6 / 10$ & 0.76 \\
\hline $0.01 / 0.05(\Xi)$ & $\infty$ & $0.9660(40)$ & $7-18$ & $9.4 / 8$ & 0.31 \\
\hline $0.02(\mathrm{~N})$ & 0.02 & $0.8450(40)$ & $8-20$ & $16 / 9$ & 0.074 \\
\hline $0.02 / 0.05(\Lambda)$ & 0.02 & $0.9100(30)$ & $7-21$ & $16 / 11$ & 0.14 \\
\hline $0.02 / 0.05(\Xi)$ & 0.02 & $0.9752(23)$ & $7-22$ & $17 / 12$ & 0.15 \\
\hline $0.4(\mathrm{~N})$ & 0.4 & $2.4213(19)$ & $18-31$ & $4.6 / 10$ & 0.92 \\
\hline $0.2(\mathrm{~N})$ & 0.2 & $1.7075(22)$ & $16-31$ & $17 / 12$ & 0.15 \\
\hline $0.1(\mathrm{~N})$ & 0.1 & $1.3110(40)$ & $14-28$ & $17 / 11$ & 0.12 \\
\hline $0.05(\mathrm{~N})$ & 0.05 & $1.0570(50)$ & $12-25$ & $5 / 10$ & 0.89 \\
\hline $0.04(\mathrm{~N})$ & $0.04 / 0.05$ & $1.0030(30)$ & $10-22$ & $17 / 9$ & 0.044 \\
\hline $0.03(\mathrm{~N})$ & $0.03 / 0.05$ & $0.9300(27)$ & $9-22$ & $7.5 / 10$ & 0.68 \\
\hline $0.02(\mathrm{~N})$ & $0.02 / 0.05$ & $0.8540(30)$ & $8-20$ & $6.9 / 9$ & 0.65 \\
\hline $0.01(\mathrm{~N})$ & $0.01 / 0.05$ & $0.7790(60)$ & $7-16$ & $1.1 / 6$ & 0.98 \\
\hline $0.04 / 0.05(\Lambda)$ & $0.04 / 0.05$ & $1.0240(30)$ & $9-22$ & $9 / 10$ & 0.53 \\
\hline $0.03 / 0.05(\Lambda)$ & $0.03 / 0.05$ & $0.9784(23)$ & $8-21$ & $7 / 10$ & 0.72 \\
\hline $0.02 / 0.05(\Lambda)$ & $0.02 / 0.05$ & $0.9312(27)$ & $7-20$ & $9.9 / 10$ & 0.45 \\
\hline $0.01 / 0.05(\Lambda)$ & $0.01 / 0.05$ & $0.8850(50)$ & $7-18$ & $4.2 / 8$ & 0.84 \\
\hline $0.04 / 0.05(\Xi)$ & $0.04 / 0.05$ & $1.0440(30)$ & $9-22$ & $8.3 / 10$ & 0.6 \\
\hline $0.03 / 0.05(\Xi)$ & $0.03 / 0.05$ & $1.0214(20)$ & $8-22$ & $8.3 / 11$ & 0.68 \\
\hline $0.02 / 0.05(\Xi)$ & $0.02 / 0.05$ & $0.9989(20)$ & $7-21$ & $9.4 / 11$ & 0.58 \\
\hline $0.01 / 0.05(\Xi)$ & $0.01 / 0.05$ & $0.9798(27)$ & $7-20$ & $6.5 / 10$ & 0.77 \\
\hline
\end{tabular}

TABLE VIII. Octet baryon masses. The format is the same as Table III. 


\begin{tabular}{|llllll|}
\hline \hline$a m_{\text {valence }}$ & $a m_{\text {sea }}$ & $a m_{\Delta}$ & range & $\chi^{2} / D$ & conf. \\
\hline $0.4(\Delta)$ & $\infty$ & $2.424(1)$ & $10-25$ & $39 / 12$ & 0.0001 \\
$0.2(\Delta)$ & $\infty$ & $1.737(2)$ & $9-25$ & $30 / 13$ & 0.0053 \\
$0.1(\Delta)$ & $\infty$ & $1.373(6)$ & $8-17$ & $6.9 / 6$ & 0.33 \\
$0.05(\Delta)$ & $\infty$ & $1.184(12)$ & $7-13$ & $0.37 / 3$ & 0.95 \\
$0.03(\Delta)$ & $\infty$ & $1.092(12)$ & $5-11$ & $3.5 / 3$ & 0.32 \\
$0.02(\Delta)$ & $\infty$ & $1.058(15)$ & $4-9$ & $0.85 / 2$ & 0.65 \\
$0.01(\Delta)$ & $\infty$ & $1.008(17)$ & $3-7$ & $1.7 / 1$ & 0.19 \\
\hline $0.02(\Delta)$ & 0.02 & $0.991(18)$ & $4-10$ & $1.7 / 3$ & 0.65 \\
\hline $0.4(\Delta)$ & 0.4 & $2.450(6)$ & $18-25$ & $6.2 / 4$ & 0.19 \\
$0.2(\Delta)$ & 0.2 & $1.753(2)$ & $9-23$ & $12 / 11$ & 0.33 \\
$0.1(\Delta)$ & 0.1 & $1.396(6)$ & $8-17$ & $11 / 6$ & 0.08 \\
$0.05(\Delta)$ & 0.05 & $1.155(8)$ & $7-15$ & $6.4 / 5$ & 0.27 \\
$0.04(\Delta)$ & $0.04 / 0.05$ & $1.102(9)$ & $6-14$ & $8.9 / 5$ & 0.11 \\
$0.03(\Delta)$ & $0.03 / 0.05$ & $1.059(6)$ & $5-13$ & $5.8 / 5$ & 0.32 \\
$0.02(\Delta)$ & $0.02 / 0.05$ & $0.989(7)$ & $4-11$ & $10 / 4$ & 0.04 \\
$0.01(\Delta)$ & $0.01 / 0.05$ & $0.921(12)$ & $3-9$ & $8.8 / 3$ & 0.032 \\
\hline \hline
\end{tabular}

TABLE IX. Decuplet baryon masses. The format is the same as Table $\overline{\mathbb{I I I}}$.

\begin{tabular}{|lllll}
\hline \hline$a m_{\text {valence }}$ & $a m_{\text {sea }}$ & $c_{\pi}(0,0,1)$ & $c_{\pi}(0,1,1)$ & $c_{\rho}(0,0,1)$ \\
\hline 0.10 & $\infty$ & $0.981(6)$ & $0.971(5)$ & $0.984(49)$ \\
0.02 & $\infty$ & $1.001(10)$ & $0.973(21)$ & $0.900(82)$ \\
0.01 & $\infty$ & $0.996(14)$ & $0.981(59)$ & $1.021(122)$ \\
\hline 0.02 & 0.02 & $0.997(11)$ & $0.986(16)$ & $1.013(62)$ \\
\hline 0.4 & 0.4 & $0.966(19)$ & na & $0.949(16)$ \\
0.2 & 0.2 & $0.958(8)$ & $0.952(4)$ & $0.921(30)$ \\
0.1 & 0.1 & $0.982(5)$ & $0.976(5)$ & $0.954(32)$ \\
0.05 & 0.05 & $0.996(6)$ & $0.988(9)$ & $0.952(44)$ \\
0.04 & $0.04 / 0.05$ & $1.000(8)$ & $0.972(9)$ & $0.926(51)$ \\
0.03 & $0.03 / 0.05$ & $0.988(5)$ & $0.978(9)$ & $0.903(28)$ \\
0.02 & $0.02 / 0.05$ & $1.001(8)$ & $0.993(16)$ & $0.982(60)$ \\
0.01 & $0.01 / 0.05$ & $0.995(17)$ & $0.986(30)$ & $0.967(63)$ \\
\hline \hline
\end{tabular}

TABLE X. "Speed of light" for the pion and rho at $a \approx 0.13 \mathrm{fm}$. The three columns are for the Goldstone pion with momenta $\frac{2 \pi}{L}(0,0,1)$ and $\frac{2 \pi}{L}(0,1,1)$ and the $\gamma_{z} \otimes \gamma_{z}$ rho with momentum $\frac{2 \pi}{L}(0,0,1)$. 